\title{
Hopf-Frobenius Algebras and a Simpler Drinfeld Double
}

\author{
Joseph Collins ${ }^{1} \quad$ Ross Duncan ${ }^{1,2}$ \\ joseph.collins@strath.ac.uk ross.duncan@strath.ac.uk \\ ${ }^{1}$ Department of Computer and Information Sciences \\ University of Strathclyde \\ 26 Richmond Street, Glasgow, United Kingdom \\ ${ }^{2}$ Cambridge Quantum Computing Ltd \\ 9a Bridge Street, Cambridge, United Kingdom
}

\begin{abstract}
The zX-calculus and related theories are based on so-called interacting Frobenius algebras, where a pair of $\dagger$-special commutative Frobenius algebras jointly form a pair of Hopf algebras. In this setting we introduce a generalisation of this structure, Hopf-Frobenius algebras, starting from a single Hopf algebra which is not necessarily commutative or cocommutative. We provide a few necessary and sufficient conditions for a Hopf algebra to be a Hopf-Frobenius algebra, and show that every Hopf algebra in $\mathbf{F V e c t}_{\mathbf{k}}$ is a Hopf-Frobenius algebra. In addition, we show that this construction is unique up to an invertible scalar. Due to this fact, Hopf-Frobenius algebras provide two canonical notions of duality, and give us a "dual" Hopf algebra that is isomorphic to the usual dual Hopf algebra in a compact closed category. We use this isomorphism to construct a Hopf algebra isomorphic to the Drinfeld double, but has a much simpler presentation.
\end{abstract}

\section{Introduction}

In the monoidal categories approach to quantum theory [1, 13] Hopf algebras [32 have a central role in the formulation of complementary observables [12. In this setting, a quantum observable is represented as special commutative $†$-Frobenius algebra; a pair of such observables are called strongly complementary if the algebra part of the first and the coalgebra part of the second jointly form a Hopf algebra. In abstract form, this combination of structures has been studied under the name "interacting Frobenius algebras" [16] where it is shown that relatively weak commutation rules between the two Frobenius algebras produce the Hopf algebra structure. From a different starting point Bonchi et al [7] showed that a distributive law between two Hopf algebras yields a pair of Frobenius structures, an approach which has been generalised to provide a model of Petri nets [6]. Given the similarity of the two structures it is appropriate to consider both as exemplars of a common family of Hopf-Frobenius algebras.

In the above settings, the algebras considered are both commutative and cocommutative. However more general Hopf algebras, perhaps not even symmetric, are a ubiquitous structure in mathematical physics, finding applications in gauge theory [27], topological quantum field theory [3] and topological quantum computing [8]. In this paper we take the first steps towards generalising the concept of Hopf-Frobenius algebra to the non-commutative case, and opening the door to applications of categorical quantum theory in other areas of physics.

Loosely speaking, a Hopf-Frobenius algebra consists of two monoids and two comonoids such that one way of pairing a monoid with a comonoid gives two Frobenius algebras, and the other pairing yields two Hopf algebras, with the additional condition that antipodes are constructed

Bob Coecke and Mathew Leifer (Eds.):

Quantum Physics and Logic 2019 (QPL)

EPTCS 318, 2020, pp. 150-180 doi $10.4204 /$ EPTCS.318.10

(c) J. Collins \& R. Duncan 


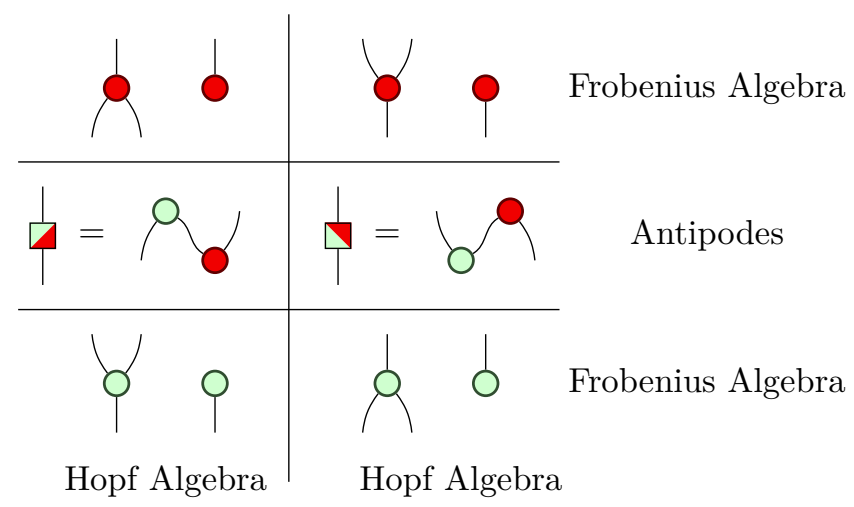

Figure 1: The elements of a Hopf-Frobenius algebra

from the Frobenius forms. This schema is illustrated in Figure1 1 In Section 3 we give the precise definition of Hopf-Frobenius algebras and state the necessary and sufficient conditions to extend a Hopf algebra to a Hopf-Frobenius algebra in an arbitrary symmetric monoidal category. It was previously known that in $\mathbf{F V e c t}_{\mathbf{k}}$, the category of finite dimensional vector spaces, every Hopf algebra carries a Frobenius algebra on both its monoid [26] and its comonoid [14, 24]; in fact every Hopf algebra in FVect $_{\mathbf{k}}$ is Hopf-Frobenius. In Section 4 we briefly present some examples which are not the usual abelian group algebras. In Section 5 we show the structure of a Hopf-Frobenius algebra can be used to give a simpler version of the Drinfeld double construction.

Acknowledgements The authors wish to thank Dr Gabriella Böhm (Wigner Research Centre for Physics) for her very kind email, and all of the help and input that she gave us. The authors also wish to thank the anonymous reviewer for their many useful and insightful comments. Joseph Collins is supported by a Carnegie Trust PhD Scholarship.

\section{Preliminaries}

We assume that the reader is familiar with strict symmetric monoidal categories and their diagrammatic notation; see Selinger [30] for a thorough treatment. We make the convention that diagrams are read from top to bottom. When we work with the dual of an object, we will opt to omit the object names from the wires except where doing so would create ambiguity. Instead, we will assign an orientation to the wires: downwards for the original object, upwards for its dual.

Definition 2.1. In a monoidal category $\mathcal{C}$ with objects $A$ and $B, B$ is left dual to $A$ if there exists morphisms $d: I \rightarrow A \otimes B$ and $e: B \otimes A \rightarrow I$ such that

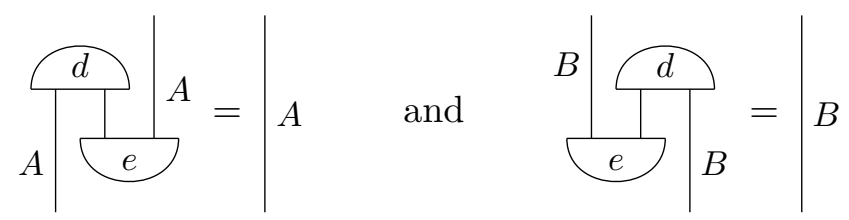

In this circumstance $A$ is right dual to $B$. Note that if $\mathcal{C}$ is symmetric then left duals and right duals coincide. 
The morphisms $d$ and $e$ are usually called the unit and counit; for reasons which will become obvious shortly we avoid that terminology and refer to them as the cap and the cup. Note that if an object has a dual it is unique up to isomorphism (see Lemma C.1).

Definition 2.2. A compact closed category [22] is a symmetric monoidal category where every object $A$ has an assigned dual $\left(A^{*}, d_{A}, e_{A}\right)$. In the graphical notation we depict the cup and cap in the obvious way:

$$
d_{A}:=A \uparrow A^{*} \quad e_{A}:=A^{*} \uparrow \downarrow^{*}
$$

Proposition $2.3([22])$. Let $\mathcal{C}$ be a compact closed category. By defining $f^{*}: B^{*} \rightarrow A^{*}$ as

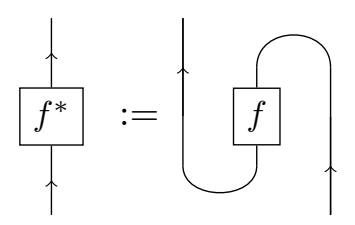

the assignment of duals $A \mapsto A^{*}$ extends uniquely to a strong monoidal functor $(\cdot)^{*}: \mathcal{C}^{\mathrm{op}} \rightarrow \mathcal{C}$, with natural isomorphisms $(A \otimes B)^{*} \cong B^{*} \otimes A^{*}, A^{* *} \cong A$, and $I^{*} \cong I$ and, further, $d$ and $e$ are natural transformations.

Remark 2.4. Note that if $A$ is its own dual, a further collection of coherence equations must apply; see Selinger [29].

The main foci of this work - Frobenius and Hopf algebras - combine the structure of a monoid and a comonoid on the same object. See Appendix C.2 for basic definitions.

Definition 2.5. A Frobenius algebra in a symmetric monoidal category $\mathcal{C}$ consists of a monoid and a comonoid on the same object, obeying the Frobenius law, shown below on the left:
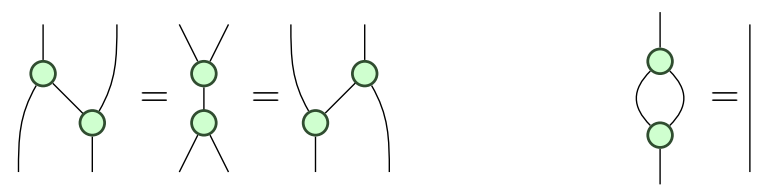

A Frobenius algebra is called special or separable when it obeys the equation above right, and quasi-special when it obeys the special equation up to an invertible scalar factor. A Frobenius algebra is commutative when its monoid is, and cocommutative when its comonoid is.

Lemma 2.6. Every Frobenius algebra induces a cup and a cap which make the object self-dual.

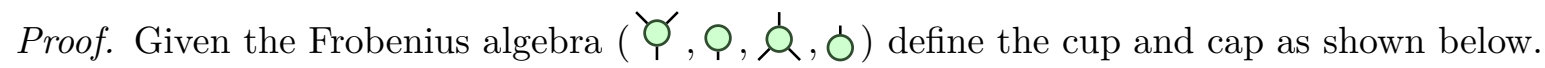

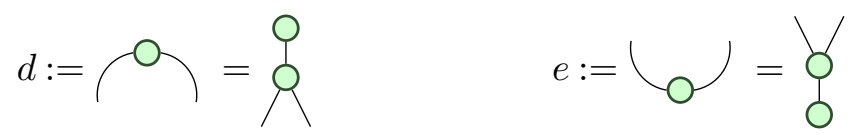

From here the snake equation follows directly.

Definition 2.5, due to Carboni and Walters [9], has a pleasing symmetry between the monoid and comonoid parts. However, an older equivalent definition will be useful in later sections 1

\footnotetext{
${ }^{1}$ See Fauser's survey [17] for several equivalent definitions.
} 
Definition 2.7. A Frobenius algebra in a symmetric monoidal category $\mathcal{C}$ consists of a monoid $\left(F,{ }_{\mathrm{Y}}, \mathrm{Q}\right)$ and a Frobenius form $\beta: F \otimes F \rightarrow I$, which admits an inverse, $\bar{\beta}: I \rightarrow F \otimes F$, satisfying:
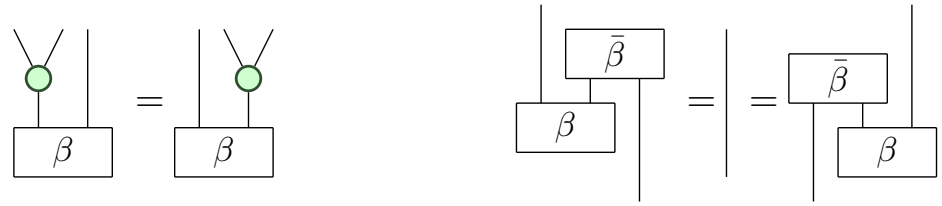

To see that Definition 2.5 implies this definition it suffices to take the cup and cap defined above as $\beta$ and $\bar{\beta}$. For the converse, we dualise ${ }^{\prime}$ with $\beta$ to get a comonoid. For a proof of how this comonoid fulfills the Frobenius law, see Kock [23]

Frobenius forms are far from unique: there is one for each invertible element of the monoid (see Appendix C.3).

Special Frobenius algebras can be understood as arising from a distribution law of comonoids over monoids [25]. In the other direction, distributing monoids over comonoids yields bialgebras.

Note. Unlike the preceding section, in our discussion of bialgebras and Hopf algebras, we will use different colours for the monoid and comonoid parts of the structure.

Definition 2.8. A bialgebra in symmetric monoidal category $\mathcal{C}$ consists of a monoid and a comonoid on the same object, which jointly obey the copy, cocopy, bialgebra, and scalar laws depicted below.
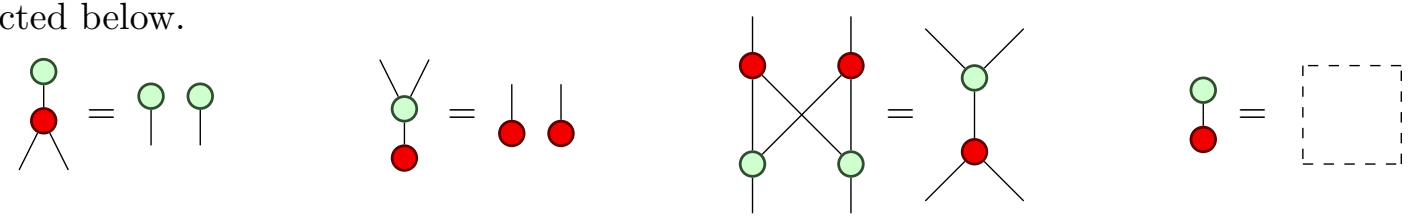

Note that the dashed box above represents an empty diagram. We may equivalently define a bialgebra as a monoid and a comonoid such that the comonoid is a monoid homomorphism. A bialgebra morphism is an morphism of the object which is both a monoid homomorphism and a comonoid homomorphism.

Remark 2.9. Some works, notably on the zX-calculus [12, 2, 20] and related theories [16], the last axiom is dropped and the other equations modified by a scalar factor, to give a scaled bialgebra. Here we use the standard definition: the Frobenius algebras we construct will not be special.

Definition 2.10. A Hopf algebra consists of a bialgebra $\left(H,{ }^{\mathbf{Y}}, \mathrm{Q}, \mathbf{\phi}, \mathbf{\phi}\right)$ and an endomorphism $s: H \rightarrow H$ called the antipode which satisfies the Hopf law:

$$
s:=
$$

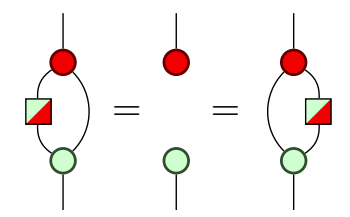

Where unambiguous, we abuse notation slightly and use $H$ to refer the whole Hopf algebra. Following Street [31, we can define another Hopf algebra $H^{\mathrm{op}}$ on the same object, having the same unit and counit, but with the arguments of the multiplication and comultiplication swapped:
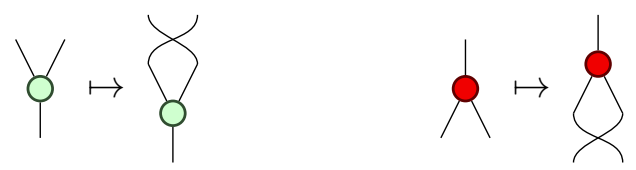
Replacing only the comultiplication as above yields a bialgebra $H^{\sigma}$ which is not necessarily Hopf. We quote the following basic properties from Street [31].

Proposition 2.11. For a Hopf algebra $H$ :

1. The antipode $s$ is unique.

2. $s: H^{\mathrm{op}} \rightarrow H$ is a bialgebra homomorphism, i.e.

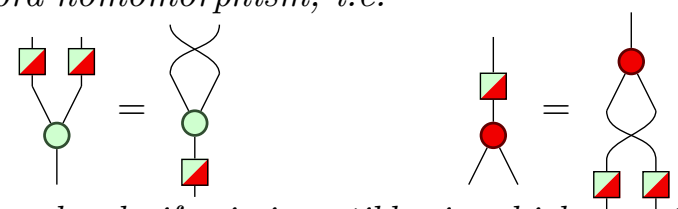

3. $H^{\sigma}$ is a Hopf algebra if and only if $s$ is invertible, in which case the antipode of $H^{\sigma}$ is $s^{-1}$.

4. If $H$ is commutative or cocommutative then $s \circ s=\mathrm{id}_{H}$.

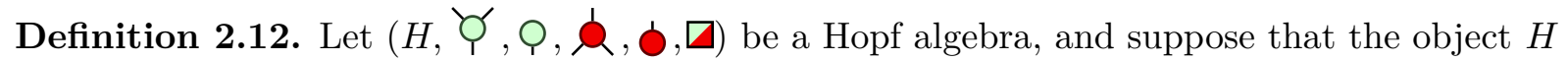
has a left dual $H^{*}$. We define the dual Hopf algebra $\left(H^{*}, \mathbf{Q}^{*}, \mathbf{O}^{*}, \mathbf{Y}^{*}, \mathbf{Q}^{*}, \mathbf{\square}^{*}\right)$ as :

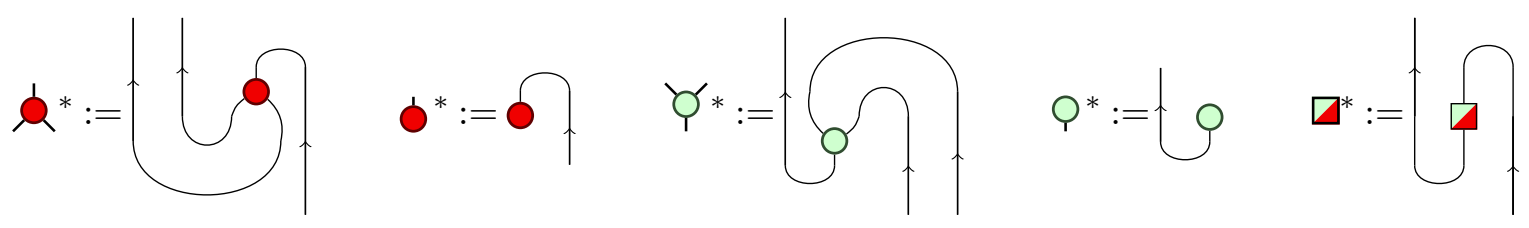

It's straightforward to prove that $H^{*}$ is indeed a Hopf algebra using the equations of Def 2.1 In later sections it will be helpful to consider duals with respect to different cups and caps, in which case we will vary notation accordingly but the same construction is used in all cases.

\section{Hopf-Frobenius Algebras}

We now arrive at the main subject of this paper, Hopf-Frobenius algebras in an arbitrary symmetric monoidal category $\mathcal{C}$. These algebras generalise interacting Frobenius algebras [12, 16], and share the same gross structure. It will be helpful to introduce a weaker notion first.

Definition 3.1. A pre-Hopf-Frobenius algebra or pre-HF algebra consists of an object $H$ bearing

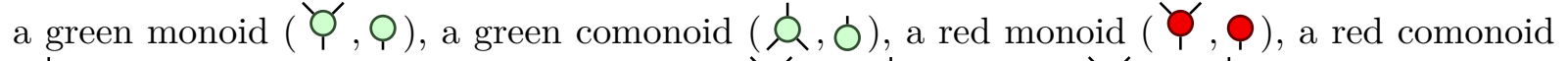

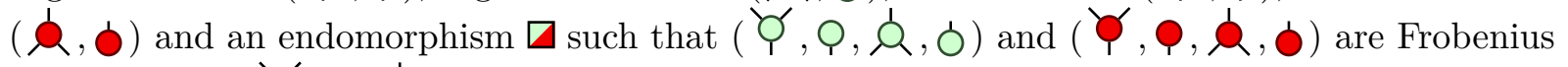
algebras, and ( $\boldsymbol{Y}, \mathrm{Q}, \boldsymbol{\phi}, \boldsymbol{\phi}, \boldsymbol{\nabla})$ is a Hopf algebra.

Definition 3.2. A Hopf-Frobenius algebra, or HF algebra, is a pre-Hopf-Frobenius algebra where $\square$ satisfies the left equation below,

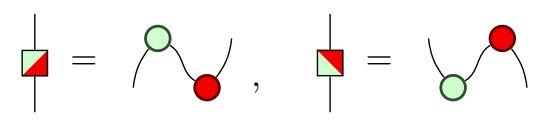

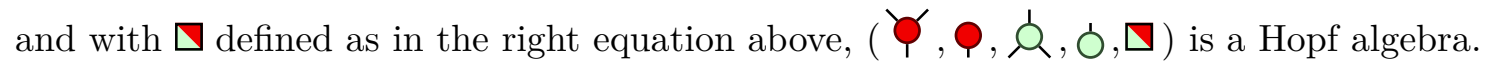

We refer to the four algebras that make up an HF algebra by the coloun $2^{2}$ of their multiplication,

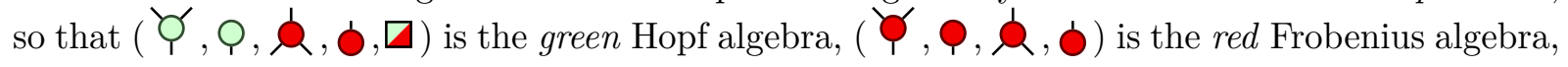
etc.

\footnotetext{
${ }^{2}$ If you are reading this document in monochrome green will appear as light grey and red as dark grey.
} 
We now move on to the main topic of the section: under what conditions does a Hopf algebra extend to a Hopf-Frobenius algebra? Henceforward, unless otherwise stated, $\mathcal{C}$ will denote a symmetric monoidal category, and $H$ will denote a $\operatorname{Hopf}$ algebra $(H, \stackrel{\mathrm{Y}}{,}, \mathrm{Q}, \boldsymbol{\mathbf { Q }}, \boldsymbol{\mathbf { O }}, \boldsymbol{\square})$ in $\mathcal{C}$. Omitted proofs are found in Appendix A.

A key concept is that of an integral. Pareigis 28 proved ${ }^{3}$ that in $\mathbf{F P M o d}_{\mathbf{R}}$, the category of finitely generated projective modules over a commutative ring, a Hopf algebra has Frobenius structure when its space of integrals is isomorphic to the ring. More generally, Takeuchi [34] and Bespalov et al. [5] gave conditions for the space of integrals in certain braided monoidal categories to be invertible.

Definition 3.3. A left (co)integral on $H$ is a copoint $\downarrow: H \rightarrow I$ (resp. a point $\left.{ }^{\star}: I \rightarrow H\right)$, satisfying the equations:

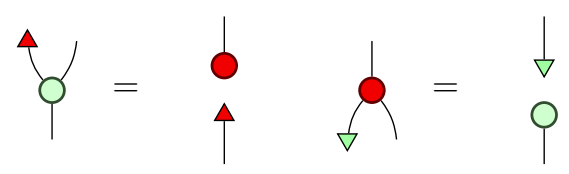

A right (co)integral is defined similarly.

Definition 3.4. An integral Hopf algebra $(H, \vec{\uparrow}, \nabla)$ is a Hopf algebra $H$ equipped with a choice of right integral $\vec{\nabla}$, and left cointegral $\uparrow$, such that $\dot{\nabla} \circ \uparrow=\mathrm{id}_{I}$.

Lemma 3.5. Let $(H, \uparrow, \nabla)$ be an integral Hopf algebra. Then the following map is the inverse of the antipode.

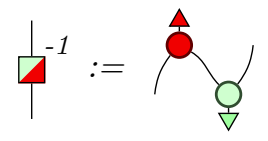

The statement of the above Lemma bares some similarities with Definition 2.1. In what follows, we will be generalising this definition to capture the situation that arises with integral Hopf algebras.

Definition 3.6. Let $A$ and $B$ be objects in a symmetric monoidal category $\mathcal{C}$. A is a right half dual of $B$ if there exists morphisms $\cap: I \rightarrow A \otimes B$ and $\forall: B \otimes A \rightarrow I$ which satisfy the following equation

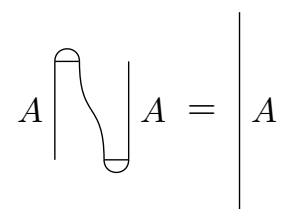

In this circumstance, $B$ is a left half dual of $A$

Half duals are a strict generalisation of duals in the sense of Definition 2.1. Unlike true duals, an object may have non-isomorphic half duals. For example, if $B$ is left dual to $A$, with a section $m: B \hookrightarrow C$ for some retraction $m^{\prime}: C \rightarrow B$, then $C$ is a left half dual of $A$. Further, any integral Hopf algebra $(H, \uparrow, \downarrow)$ makes $H$ left half dual to itself as follows.

Definition 3.7. Let $(H, \uparrow, \nabla)$ be an integral Hopf algebra, and define

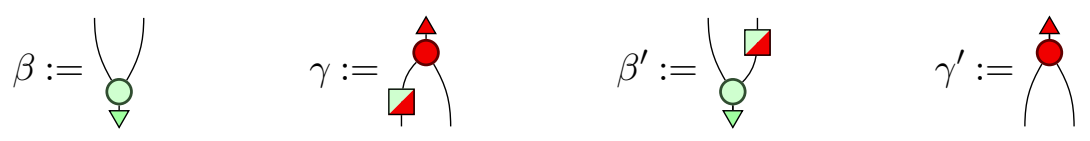

\footnotetext{
${ }^{3}$ This is a generalisation of earlier work by Larson and Sweedler [26] showing that the space of integrals in FVect $_{\mathbf{k}}$ is always isomorphic to $k$.
} 
With these definitions, $\gamma$ and $\beta$ make $H$ half dual to itself, and $\gamma^{\prime}$ and $\beta^{\prime}$ make $H$ half dual to itself but in a different way. We say that $H$ is nondegenerate when $\gamma$ and $\beta$ are a cap and a cup respectively, (c.f. Definition 2.2), making $H$ fully dual to itself. Furthermore, in this situation, $\gamma^{\prime}$ and $\beta^{\prime}$ are also a cup and a cap, giving a different self-dual structure to $H$.

Lemma 3.8. Let $(H, \uparrow, \nabla)$ be an integral Hopf algebra. $H$ is nondegenerate if and only if

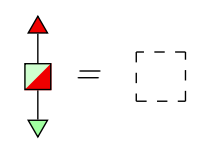

Lemma 3.9. Let $(H, \uparrow, \dot{\nabla})$ be an integral Hopf algebra. $H$ is nondegenerate if and only if $\beta$ is a Frobenius form for $\left(H,{ }^{\prime}, Q\right.$, ) or equivalently, if and only if $\gamma^{\prime}$ is a Frobenius form for $(H, \mathbf{Q}, \mathbf{O})$. Hence, if $H$ is nondegenerate, then $H$ admits a pre-HF algebra structure.

Per Definition 2.7. $\nabla$ is the counit of the green Frobenius algebra and the green comultiplication is obtained by dualising ${ }_{\mathrm{Y}}^{\mathrm{Y}}$ with $\beta$. The red unit and multiplication are obtained in a similar manner.

Definition 3.10. Let the object $H$ have a right half dual $H^{*}$. The integral morphism $\mathcal{I}: H \rightarrow H$ is defined as shown below.

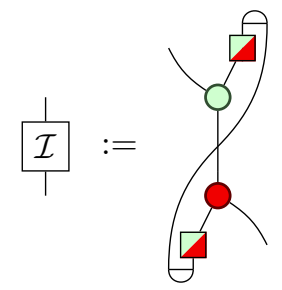

Note that this definition does not depend on the choice of half dual - see Lemma A.2

If $H$ is in FPMod $_{\mathbf{R}}$, then $\mathcal{I}$ may be seen as a map from $H$ to the space of left cointegrals. In fact, it is the retraction of the natural injection from the space of left integrals into $H$. As such, it acts trivially on integrals, and for every element $v \in H, \mathcal{I}(v)$ is a left integral (which may be 0). In Lemma 3.11 we show that this holds in the general case, where we have exchanged elements of a module for points $p: I \rightarrow H$.

Lemma 3.11. Given a point $p: I \rightarrow H$, and copoint $q: H \rightarrow I$, the morphism $\mathcal{I} \circ p$ is a left cointegral, and $q \circ \mathcal{I}$ is a right integral. In addition, $p$ is a left cointegral if and only if $\mathcal{I} \circ p=p$, and $q$ is a right integral if and only if $q \circ \mathcal{I}=q$.

Definition 3.12. We say that a Hopf algebra satisfies the Frobenius condition if there exists maps $\boldsymbol{\phi}$ and $\nabla$ such that

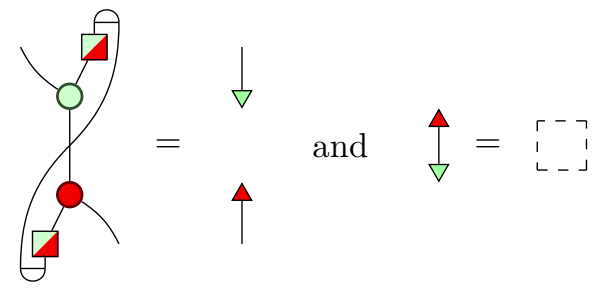


Theorem 3.13. If $H$ satisfies the Frobenius condition, then $H$ admits a pre-HF algebra structure with the Frobenius forms and their inverses as shown below.<smiles>COC=COC</smiles><smiles>COC=COC</smiles>
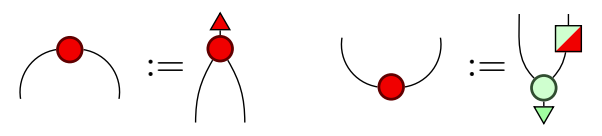

Further, $(H, \uparrow, \nabla)$ is an integral Hopf algebra.

Proof. The Frobenius condition implies that $\mathcal{I} \circ \hat{\boldsymbol{\top}}=\boldsymbol{\uparrow}$, and $\nabla \circ \mathcal{I}=\dot{\nabla}$. Hence, by Lemma 3.11, $\boldsymbol{\uparrow}$ is a left cointegral and $\downarrow$ is a right integral. Now, we only need to show that it is nondegenerate. Observe that

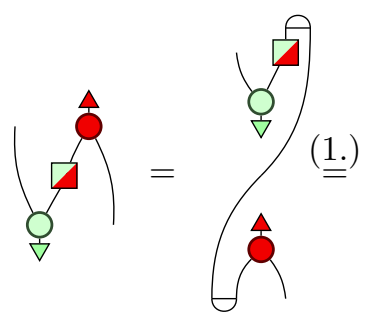

$(\underline{\underline{4}})$

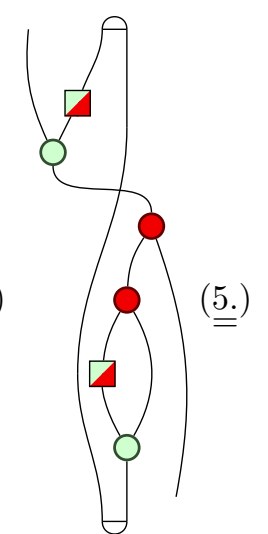

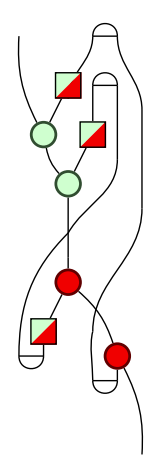

$\underline{\underline{2} .})$

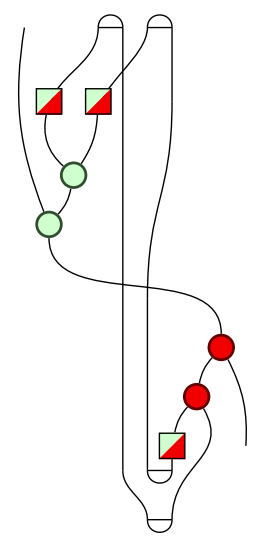

(3.)

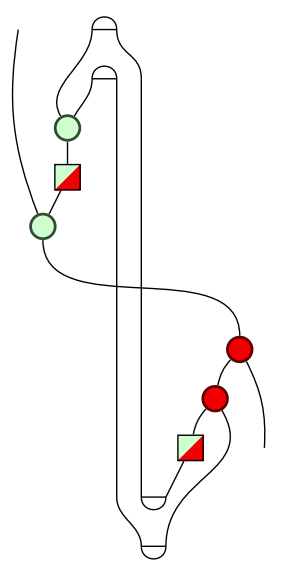

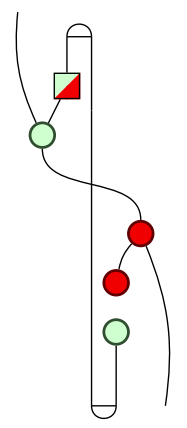

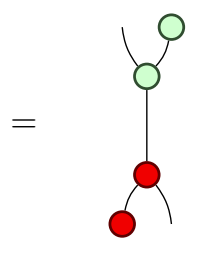

where (1.) is due to the Frobenius condition, (2.) comes from associativity and (3.) comes from the fact that the antipode is a bialgebra homomorphism $H^{\mathrm{op}} \rightarrow H$. The presence of half duals gives us (4.), and (5.) is due to the Hopf law. We then get the following identity

$$
\left.\left.\right|_{0} ^{\infty}\right|^{\circ}=
$$

This is the identity required to make $(H, \uparrow, \downarrow)$ nondegenerate, and we have our result by Lemma 3.9 . 
The explicit definitions of the green comonoid and red monoid structures are shown below.

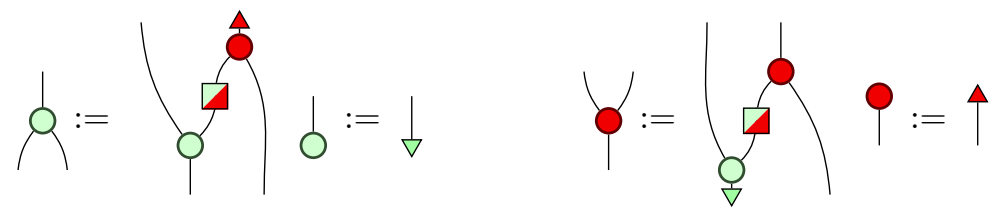

As the name suggests, $H$ fulfilling the Frobenius condition is equivalent to $H$ admitting a Frobenius algebra structure. To prove this, we must first prove the following intermediate lemma

Lemma 3.14. Let the object $H$ have a right half dual $H^{*}$, where $H$ is a Hopf algebra. $H$ fulfills the Frobenius condition if and only if there is an equaliser 4 of

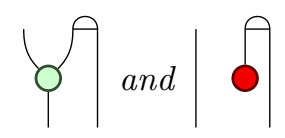

if and only if there is a coequaliser $\nabla$ of

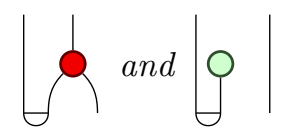

Theorem 3.15. Let $H$ be a Hopf algebra. $H$ satisfies the Frobenius condition if and only if $H$ admits a Frobenius structure on its multiplication or its comultiplication. Hence, $H$ fulfills the Frobenius condition if and only if it admits a pre-HF algebra structure.

Proof. Clearly if $H$ satisfies the Frobenius condition, then by Theorem 3.13 it admits a Frobenius structure. For the converse, suppose that $H$ admits a Frobenius structure $(H, \mathcal{Y}, \mathbf{Q}, \mathbf{Q}, \boldsymbol{O})$ on its multiplication. This provides a cup and a cap that makes $H$ self dual. Set $\alpha:=\}$. We will show that $\alpha: I \rightarrow H$ is a split equaliser of the diagram

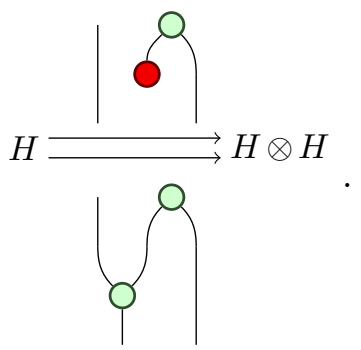

Note that the lower morphism is simply $\alpha$. To show that $\alpha$ is a split equaliser, we must first show that it is is a cone of the appropriate diagram. This follows from the properties of the Frobenius algebra

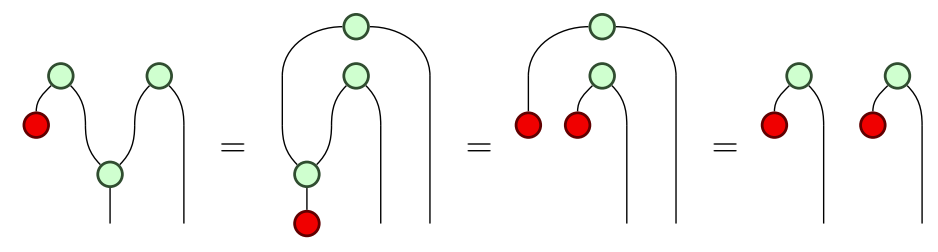


We now need to find a retract of $\alpha$ and $\alpha$. It is clear that $\operatorname{Ois}$ a retract of $\alpha$, and $\downarrow \mid$ is a retract of $\alpha$. The final condition for $\alpha$ to be a split equaliser is

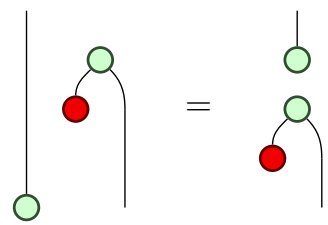

Thus, $\alpha$ a split equaliser. By Lemma 3.14, this implies that $H$ must satisfy the Frobenius condition. If $H$ admits a Frobenius algebra on its comultiplication, then the same result holds by duality. Hence, when $H$ admits a pre-HF algebra structure, it fulfills the Frobenius condition, and by Theorem 3.13 , we get our equivalence.

It may be important to mention that if $H$ admits a Frobenius structure, then this is not necessarily the same structure as the one given by Theorem 3.13 . In Proposition C.8, we show that Frobenius structures are not unique. One may start with different Frobenius structures, and end up with the same pre-HF algebra. In the proof of Lemma 3.15, one constructs an integral and cointegral, and it is these that determine the appropriate Frobenius structure.

Pareigis [28] showed that in $\mathbf{F P M o d}_{\mathbf{R}}$, a Hopf Algebra will admit a Frobenius algebra when integrals only differ by a scalar multiple. This is clear from Lemma 3.14 . Under mild assumptions, this is equivalent to the Frobenius condition.

In a monoidal category, an object $A$ is said to have enough points if, for all morphisms $f, g: A \rightarrow B$, we have

$$
(\forall x: I \rightarrow A, \quad f x=g x) \Rightarrow f=g .
$$

Lemma 3.16. Let $(H, \uparrow, \nabla)$ be an integral Hopf algebra and suppose that $H$ has enough points. If every left cointegral (right integral) is a scalar multiple of $\mathbf{Q}$ (resp. O) then $H$ fulfills the Frobenius condition

Since $\mathbf{F P M o d}$ (and $\mathbf{F V e c t}_{\mathbf{k}}$ ) are categories where every object has enough points, Lemma 3.16 implies Pareigis' condition is exactly the Frobenius condition.

We may now consider the main theorem of the paper - when exactly does a Hopf algebra admit a Hopf-Frobenius algebra?

Theorem 3.17. Let $H$ be a Hopf algebra such that the object $H$ has some weak right dual $H^{*}$. Then $H$ admits a Hopf-Frobenius algebra structure if and only if $H$ fulfills the Frobenius condition.

Sketch of Proof. We explore this in full detail in the appendix. Here, we only outline a sketch of the proof

If $H$ is a Hopf-Frobenius algebra, then it admits a Frobenius algebra, and therefore, by Theorem 3.15, it fulfills the Frobenius condition.

Consider the converse. By Theorem 3.13, we know that if $H$ fulfills the Frobenius condition, then $H$ admits a pre-HF algebra and $(H, ९, \bigotimes)$ is an integral Hopf algebra. It follows from Lemma 3.5 that $\boldsymbol{\nabla}=\Omega$, and we show in Lemma A.3 that this is true if and only if $(H, \bigcirc, \bigcirc)$ is an integral Hopf algebra. Hence, $H$ admits Hopf-Frobenius structure if and 
only if $(H, \zeta, \varphi, \phi, \phi, \nabla)$ forms a Hopf algebra, where $\nabla=\gamma$. We begin by proving that $(H, \boldsymbol{\gamma}, \boldsymbol{\varphi}, \boldsymbol{Q}, \boldsymbol{O})$ is a bialgebra, and then that $\boldsymbol{\nabla}=\boldsymbol{\gamma}$ is the appropriate antipode to make this bialgebra a Hopf algebra.

$H$ admits pre-HF algebra structure, so it has a structure that makes $H$ self dual. Let $(\cdot)^{\circ}$ be the duality defined by the green Frobenius algebra. The dual of a Hopf algebra is a Hopf algebra, in the sense of Definition 2.12. Therefore, applying the dual to $H$ will give us another Hopf algebra. Lemma A.5 tells us that

$$
(\phi)^{\circ}=q \quad(q)^{\circ}=\varnothing
$$

Set $H^{\circ}:=\left(H, \mathbf{Q}^{\circ}, \mathbf{0}^{\circ}, \mathrm{\gamma}^{\circ}, \mathrm{O}^{\circ}, \mathbf{\square}^{\circ}\right)$ to be the Hopf algebra obtained when we apply $(\cdot)^{\circ}$

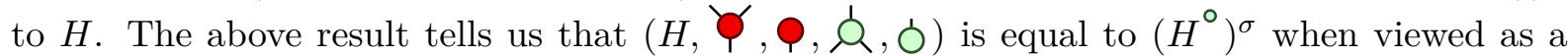
bialgebra. Therefore, by Proposition 2.11 we only need to show that $\square^{\circ}$ has an inverse. But the duality operation, $(\cdot)^{\circ}$, maps isomorphisms to isomorphisms, so since $\boldsymbol{\nabla}$ is invertible, $\boldsymbol{\nabla}:=\left(\boldsymbol{\nabla}^{-1}\right)^{\circ}$ will be the antipode of $(H, \boldsymbol{\gamma}, \boldsymbol{\varphi}, \boldsymbol{\alpha}, \boldsymbol{O})$. All that is left is to show that $\boldsymbol{\nabla}=\boldsymbol{\gamma}$, and this is accomplished by simple calculation.

Let us summarise the various equivalent conditions for a Hopf algebra to be Hopf-Frobenius.

Theorem 3.18. Let $H$ be a Hopf algebra. The following conditions are equivalent

- $H$ admits a Hopf-Frobenius algebra structure

- $H$ admits a pre-HF algebra structure

- $H$ fulfills the Frobenius condition

- H admits a Frobenius algebra structure on the multiplication or the comultiplication

- $H$ admits an equaliser $\boldsymbol{\uparrow}$ of

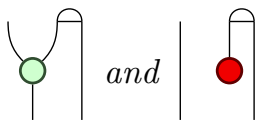

- $H$ admits an integral algebra structure, $(H, \uparrow, \nabla)$, and $H$ is nondegenerate

- $H$ admits an integral algebra structure, $(H, \uparrow, \nabla)$, and $\nabla \circ \square \circ \uparrow=1_{I}$

We finish this section by asking how canonical this structure is. Frobenius structure in general is non-canonical (cf. Proposition C.8). Despite this, we find that Hopf-Frobenius structure is canonical, as follows.

Lemma 3.19. Let $H$ admit a Hopf-Frobenius algebra structure. Then this structure is unique up to invertible scalar.

\section{Examples}

Combined with the results of Larson and Sweedler [26], Pareigis [28], and Lemma 3.16, Theorem 3.17 implies that any Hopf algebra in $\mathbf{F V e c t}_{\mathbf{k}}$ is Hopf-Frobenius. This allows the direct extension of [16] to non-abelian group algebras, but there are plenty of other examples. We briefly mention some examples which are neither commutative nor cocommutative. 
Example 4.1. Let $k$ be a field with a primitive $n^{\text {th }}$ root of unity $z$. The Taft Hopf algebras [33] are a family of Hopf algebras in FVect $_{\mathbf{k}}$ whose antipodes have order $2 n$. Generically, the algebra $(H, \mu, 1, \Delta, \epsilon, s)$ is generated by elements $x$ and $g$, such that $x^{n}=0, g^{n}=1$, and $g x=z x g$. The coalgebra is defined $\Delta(x)=1 \otimes x+x \otimes g$, and $\Delta(g)=g \otimes g$, with $\epsilon(x)=0$ and $\epsilon(g)=1$. The antipode is $s(x)=-x g^{-1}, s(g)=g$, and the rest of the structure follows from the Hopf algebra axioms. We may see that $H$ has the basis $x^{\alpha} g^{\beta}$, where $0 \leq \alpha, \beta, \leq n-1$, so this will imply that $H$ is $n^{2}$ dimensional. One can calculate that the left integral of $H$ is

$$
\sum_{i=1}^{n} z^{-i} g^{i} x^{n-1}
$$

and the right cointegral is the functional that takes $x^{n-1}$ to 1 and every other basis element to 0 . We explicitly construct the HF algebra of the Taft Hopf algebra when $n=2$ in the appendix.

Example 4.2. Hopf algebras which arise as the quantum enveloping algebra of Lie algebras are a type of quantum group. Since these are infinite dimensional, they cannot be Hopf-Frobenius algebras. However their finite dimensional quotients will be Hopf-Frobenius. See Kassel [21] for an example.

Moving away from FVect $_{\mathbf{k}}$, we consider Rel, the category of sets and relations.

Example 4.3. Let $G$ be an infinite group. Following Hasegawa [19] we can construct its group algebra in Rel. The integral is $\{(\star, g) \mid g \in G\}$ and the cointegral is the singleton $(1, \star)$. The construction detailed in Theorem 3.13 recovers the expected multiplication and comultiplication relations:

$$
\begin{gathered}
\grave{\alpha}:=a \mapsto(b, c) \text { such that } a=b c \\
\boldsymbol{\gamma}^{\prime}:=(a, b) \mapsto \begin{cases}a & \text { if } a=b \\
\emptyset & \text { otherwise }\end{cases}
\end{gathered}
$$

We look forward to discovering more exotic examples.

\section{$5 \quad$ A simpler Drinfeld double}

Braided categories of modules over a Hopf algebra are widely used in physics, where they give solutions to the Yang-Baxter equation and in low dimensional topology, where they are used to find invariants. However the category of modules over a Hopf algebra is braided if and only if the Hopf algebra is quasi-triangular. The Drinfeld double [15] is a construction that takes a Hopf algebra $H$ in $\mathbf{F V e c t}_{\mathbf{k}}$, and produces a quasi-triangular Hopf algebra $D(H)$ on the object $H \otimes H^{*}$. In this section we use the self-duality of a Hopf-Frobenius algebra to construct the canonical isomorphism $H \cong H^{*}$ and thus define a simpler version of the Drinfeld double on $H \otimes H$.

We will assume that $\mathcal{C}$ is a compact closed category. We denote the green and red Hopf algebras of $H$ as $H_{\circ}$ and $H_{\bullet}$ respectively. We use the generalisation of Drinfeld's original construction to symmetric monoidal categories, due to Chen [10].

Definition 5.1. Let $H$ be a $\mathrm{HF}$ algebra on $\mathcal{C}$. By Proposition C.1, we may define an isomorphism $\square: H \rightarrow H^{*}$, with inverse $-1: H^{*} \rightarrow H$ as

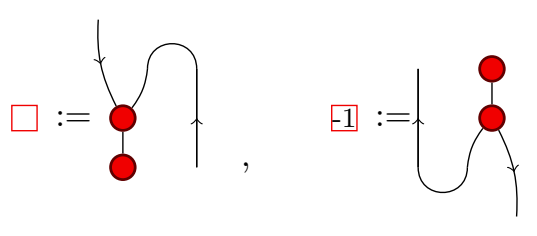


Lemma 5.2. The morphism $\square$ is a Hopf algebra homomorphism between $H_{\bullet}^{\sigma}$ and $H_{\circ}^{*}$.

Remark 5.3. The morphism $\square$ is the canonical isomorphism between the compact closed structure and the red dual structure given to us by the Hopf-Frobenius structure, in the sense of Proposition C.1. By Lemma 3.19, since the Hopf-Frobenius structure is canonical, the red and green Frobenius structures are also canonical, and by extension, the red and green dual structures on $H$ are also canonical. Therefore, whenever $H$ admits a Hopf-Frobenius structure on a compact closed category, we may construct $\square$ up to a unique invertible scalar.

Definition 5.4. A Hopf algebra $H$ is quasi-triangular if there exists a universal $R$-matrix $R: I \rightarrow H \otimes H$ such that

- $R$ is invertible with respect to ${ }^{\prime}$

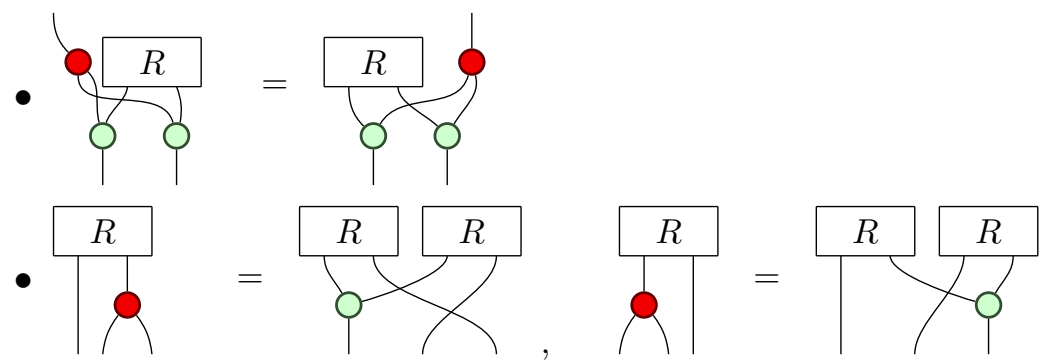

All cocommutative Hopf algebras are quasi-triangular, with $\varnothing \otimes \mathbf{0}$ as the universal $R$-matrix. This definition is motivated by the following theorem [21].

Theorem 5.5. The category of modules over a Hopf algebra is braided if and only if the Hopf algebra is quasi-triangular.

Definition 5.6. Let $H$ be a Hopf algebra in $\mathcal{C}$ with an invertible antipode. The Drinfeld double of $H$, denoted $D(H)=\left(H \otimes H^{*}, \mu, 1, \Delta, \epsilon, s\right)$, is a Hopf algebra defined in the following manner:

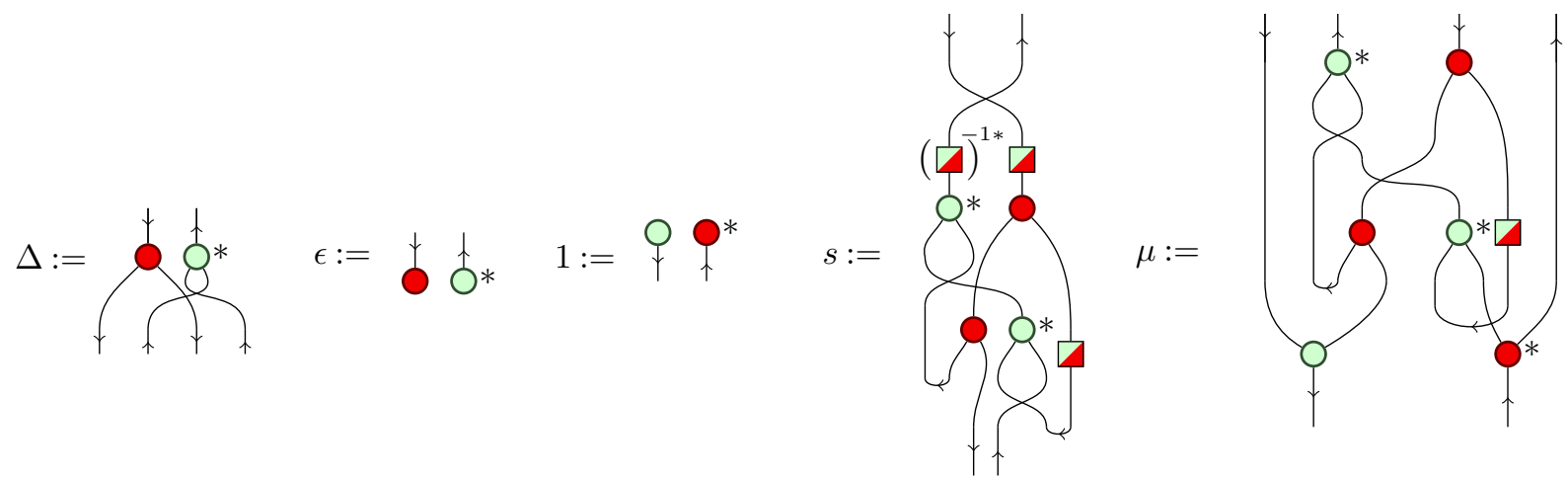

Theorem 5.7 (Drinfeld[15, 10]). D $(H)$ is quasi-triangular, with the universal $R$-matrix shown below.

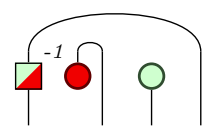

Our goal is to use the Hopf-Frobenius structure to get a Hopf algebra that is isomorphic to the Drinfeld double, but is easier to do diagrammatic reasoning with.

We will now use the Hopf-Frobenius structure to derive a Hopf algebra isomorphic to the Drinfeld double. Consider the composite of the map $1 \otimes \square$ with the multiplication of the Drinfeld double: 


\section{Lemma 5.8.}

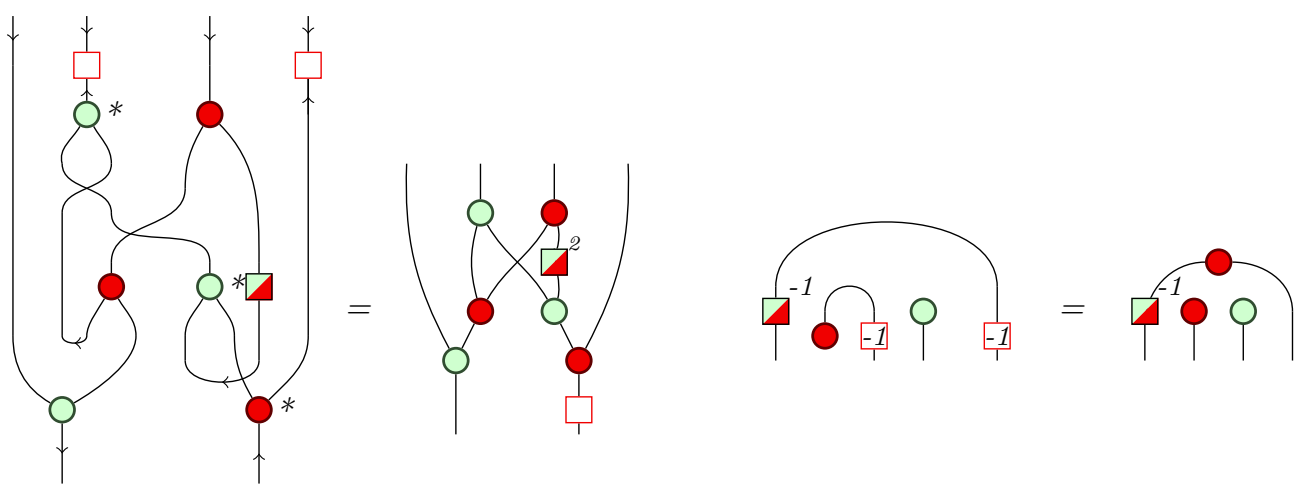

Definition 5.9. Let $H$ be a HF algebra. The red Drinfeld double, denoted $D_{\bullet}(H)=(H \otimes$ $\left.H, \mu_{\bullet}, 1_{\bullet}, \Delta_{\bullet}, \epsilon_{\bullet}, s_{\bullet}\right)$, is a Hopf algebra on the object $H \otimes H$ with structure maps

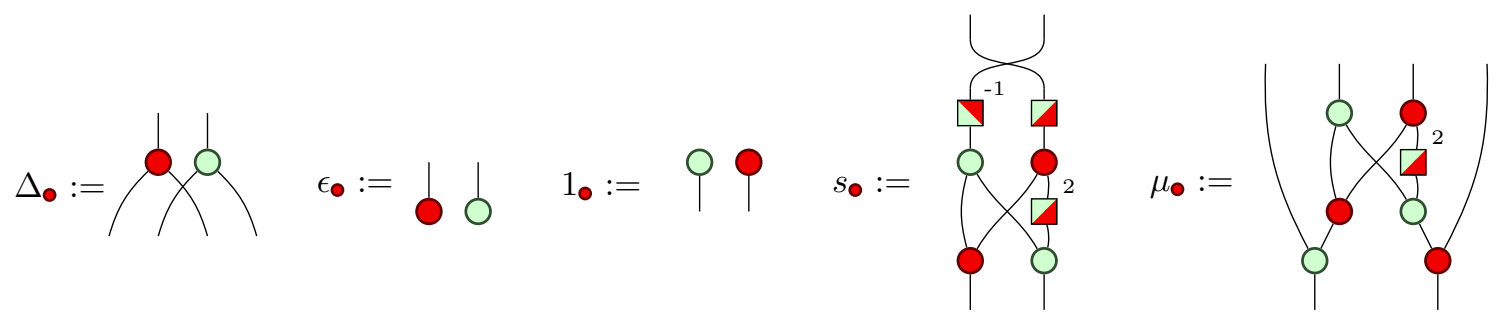

Corollary 5.10. $D_{\bullet}(H)$ is a quasi-triangular Hopf algebra isomorphic to the Drinfeld double, with universal R-matrix

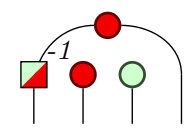

\section{Conclusion and further work}

We have generalised the notions of interacting Frobenius algebras [12, 16] and interacting Hopf algebras [7] to the non-commutative case, and in the process shown that they are rather common structures. This work could be viewed as an extension of classical results showing that concrete Hopf algebras over finite dimensional vector spaces are also Frobenius algebras [26]. Another perspective is that we make precise how much ambient symmetry is required to obtain a HopfFrobenius algebra. The original setting of interacting Frobenius algebras [12] was a $\dagger$-compact category, which provides a lot of duality on top of the commutative algebras themselves. We show that none of this structure is necessary: all that is required is one-sided half-dual for the carrier object. The major question that remains is to pin down exactly when the Frobenius condition holds; as Lemma 3.16 shows, this is tightly related to the existence of integrals. Compact closure does not suffice to guarantee this: in $\mathbf{F P M o d}_{\mathbf{R}}$ there are Hopf algebras which are not Frobenius.

While we have established that Hopf algebras are frequently Hopf-Frobenius, the resulting Frobenius algebras need not be well behaved (commutative, dagger, special) as in the original quantum setting [11. It remains to investigate what Frobenius structures arise from "interesting" Hopf algebras, and whether they have any application in the categorical quantum mechanics programme, or conversely, how HF algebras may be applied in the study of quantum groups. 
Weaker structures such as the ill-named co-Frobenius algebras or the stateful resource calculus of Bonchi et al [6] perhaps offer an alternative to the nonstandard approach [18 to study infinite dimensional systems. Beyond this, natural generalisations to the braided or planar cases suggest themselves, although this will push diagrammatic reasoning to its limits.

Our new Drinfeld double construction suggests that HF algebras could find applications in topological quantum computation, particularly for error correcting codes, an area where the ZX-calculus is already used [4]. The smallest non-abelian group is $S_{3}$, whose group algebra fits in 3 qubits with room to spare.

\section{References}

[1] S. Abramsky \& B. Coecke (2004): A categorical semantics of quantum protocols. In: Proceedings of the 19th Annual IEEE Symposium on Logic in Computer Science: LICS 2004, IEEE Computer Society, pp. 415-425, doi:10.1109/LICS.2004.1319636

[2] Miriam Backens (2015): Making the stabilizer ZX-calculus complete for scalars. In Chris Heunen, Peter Selinger \& Jamie Vicary, editors: Proceedings of the 12th International Workshop on Quantum Physics and Logic (QPL 2015), Electronic Proceedings in Theoretical Computer Science 195, pp. 17-32, doi:10.4204/EPTCS.195.2

[3] Benjamin Balsam \& Alexander Kirillov Jr (2012): Kitaev's lattice model and Turaev-Viro TQFTs. ArXiv.org. Available at https://arxiv.org/abs/1206.2308.

[4] Niel de Beaudrap \& Dominic Horsman (2020): The ZX calculus is a language for surface code lattice surgery. Quantum 4, p. 218, doi:10.22331/q-2020-01-09-218.

[5] Yuri Bespalov, Thomas Kerler, Volodymyr Lyubashenko \& Vladimir Turaev (2000): Integrals for braided Hopf algebras. Journal of Pure and Applied Algebra 148(2), pp. 113-164, doi:10.1016/S00224049(98)00169-8.

[6] Filippo Bonchi, Joshua Holland, Robin Piedeleu, PawełSobociński \& Fabio Zanasi (2019): Diagrammatic Algebra: From Linear to Concurrent Systems. Proceedings of the ACM on Programming Languages 3(POPL), pp. 25:1-25:28, doi:10.1145/3290338.

[7] Filippo Bonchi, Paweł Sobociński \& Fabio Zanasi (2017): Interacting Hopf Algebras. Journal of Pure and Applied Algebra 221(1), pp. 144-184, doi:10.1016/j.jpaa.2016.06.002.

[8] Oliver Buerschaper, Juan Martín Mombelli, Matthias Christandl \& Miguel Aguado (2013): A hierarchy of topological tensor network states. Journal of Mathematical Physics 54(1), p. 012201, doi: $10.1063 / 1.4773316$.

[9] A. Carboni \& R.F.C. Walters (1987): Cartesian bicategories I. Journal of Pure and Applied Algebra 49(1-2), doi:10.1016/0022-4049(87)90121-6.

[10] Hui-Xiang Chen (2000): Quantum doubles in monoidal categories. Communications in Algebra 28(5), pp. 2303-2328, doi:10.1080/00927870008826961

[11] B. Coecke, D. Pavlovic \& J. Vicary (2013): A new description of orthogonal bases. Math. Structures in Comp. Sci. 23(3), pp. 555-567, doi:10.1017/S0960129512000047.

[12] Bob Coecke \& Ross Duncan (2011): Interacting Quantum Observables: Categorical Algebra and Diagrammatics. New J. Phys 13(043016), doi:10.1088/1367-2630/13/4/043016 Available at http: //iopscience.iop.org/1367-2630/13/4/043016/

[13] Bob Coecke \& Aleks Kissinger (2017): Picturing Quantum Processes: A First Course in Quantum Theory and Diagrammatic Reasoning. Cambridge University Press, doi 10.1017/9781316219317.

[14] Yukio Doi \& Mitsuhiro Takeuchi (2000): Bi-Frobenius algebras. In Nicolás Andruskiewitsch, Walter Ricardo Ferrer Santos \& Hans-Jürgen Schneider, editors: New trends in Hopf algebra theory, Contemporary Mathematics 267, American Mathematical Society, pp. 67-98, doi:10.1090/conm/267/04265. 
[15] Vladimir Gershonovich Drinfeld (1986): Quantum groups. Zapiski Nauchnykh Seminarov POMI 155, pp. 18-49, doi:10.1007/BF01247086

[16] Ross Duncan \& Kevin Dunne (2016): Interacting Frobenius Algebras are Hopf. In Martin Grohe, Eric Koskinen \& Natarajan Shankar, editors: Proceedings of the 31st Annual ACM/IEEE Symposium on Logic in Computer Science, LICS '16, New York, NY, USA, July 5-8, 2016, LICS '16, ACM, pp. $535-544$, doi: $10.1145 / 2933575.2934550$.

[17] Bertfried Fauser (2013): Some Graphical Aspects of Frobenius Algebras. In Chris Heunen, Mehrnoosh Sadrzadeh \& Edward Grefenstette, editors: Quantum Physics and Linguistics: A Compositional, Diagrammatic Discourse, Oxford, doi:10.1093/acprof:oso/9780199646296.003.0002.

[18] Stefano Gogioso \& Fabrizio Genovese (2016): Infinite-dimensional Categorical Quantum Mechanics. In: Proceedings of QPL 2016, EPTCS, doi:10.4204/EPTCS.236.4

[19] Masahito Hasegawa (2010): Bialgebras in rel. Electronic Notes in Theoretical Computer Science 265, pp. 337-350, doi:10.1016/j.entcs.2010.08.020

[20] Emmanuel Jeandel, Simon Perdrix \& Renaud Vilmart (2017): A Complete Axiomatisation of the ZX-Calculus for Clifford $+T$ Quantum Mechanics. In: LICS '18- Proceedings of the 33rd Annual ACM/IEEE Symposium on Logic in Computer Science, arXiv:1705.11151, ACM, doi: $10.1145 / 3209108.3209131$.

[21] Christian Kassel (2012): Quantum groups. 155, Springer Science \& Business Media, doi:10.4171/047.

[22] G.M. Kelly \& M.L. Laplaza (1980): Coherence for Compact Closed Categories. Journal of Pure and Applied Algebra 19, pp. 193-213, doi:10.1016/0022-4049(80)90101-2.

[23] J. Kock (2003): Frobenius Algebras and 2-D Topological Quantum Field Theories. Cambridge University Press, doi:10.1017/cbo9780511615443.

[24] M. Koppinen (1996): On Algebras with Two Multiplications, Including Hopf Algebras and BoseMesner Algebras. Journal of Algebra 182(1), pp. 256 - 273, doi 10.1006/jabr.1996.0170. Available at http://www.sciencedirect.com/science/article/pii/S0021869396901702.

[25] Stephen Lack (2004): Composing PROPs. Theory and Applications of Categories 13(9), pp. 147-163.

[26] Richard Gustavus Larson \& Moss Eisenberg Sweedler (1969): An Associative Orthogonal Bilinear Form for Hopf Algebras. American Journal of Mathematics 91(1), pp. 75-94, doi $10.2307 / 2373270$. Available at https://www.jstor.org/stable/2373270

[27] Catherine Meusburger (2017): Kitaev Lattice Models as a Hopf Algebra Gauge Theory. Communications in Mathematical Physics 353(1), pp. 413-468, doi:10.1007/s00220-017-2860-7.

[28] Bodo Pareigis (1971): When Hopf algebras are Frobenius algebras. Journal of Algebra 18(4), pp. 588 - 596, doi:10.1016/0021-8693(71)90141-4. Available at http://www.sciencedirect.com/science/ article/pii/0021869371901414

[29] P. Selinger (2010): Autonomous categories in which $A \cong A^{*}$. In B. Coecke, P. Panangaden \& P. Selinger, editors: Proceedings of 7th Workshop on Quantum Physics and Logic (QPL 2010). Available at http://www.mscs.dal.ca/ selinger/papers/halftwist-2up.pdf

[30] Peter Selinger (2011): A survey of graphical languages for monoidal categories. In Bob Coecke, editor: New structures for physics, Lecture Notes in Physics 813, Springer, pp. 289-355, doi:10.1007/978-3642-12821-9_4.

[31] R. Street (2007): Quantum Groups: A Path to Current Algebra. Australian Mathematical Society Lecture Series, Cambridge University Press, doi:10.1017/CBO9780511618505

[32] Moss E. Sweedler (1969): Hopf Algebras. W. A. Benjamin Inc.

[33] Earl J. Taft (1971): The Order of the Antipode of Finite-dimensional Hopf Algebra. Proceedings of the National Academy of Sciences 68(11), pp. 2631-2633, doi:10.1073/pnas.68.11.2631. Available at https://www.pnas.org/content/68/11/2631. 
[34] Mitsuhiro Takeuchi (1999): Finite Hopf algebras in braided tensor categories. Journal of Pure and Applied Algebra 138(1), pp. 59-82, doi:10.1016/s0022-4049(97)00207-7. 


\section{A Proofs omitted from the main body of the paper}

Lemma 3.5. Let $(H, \uparrow, \nabla)$ be an integral Hopf algebra. Then the following map is the inverse of the antipode.

$$
\square^{-1}:=9_{0}^{\circ}
$$

Proof. From the definition of $\mathbf{\square}^{-1}$, we see that

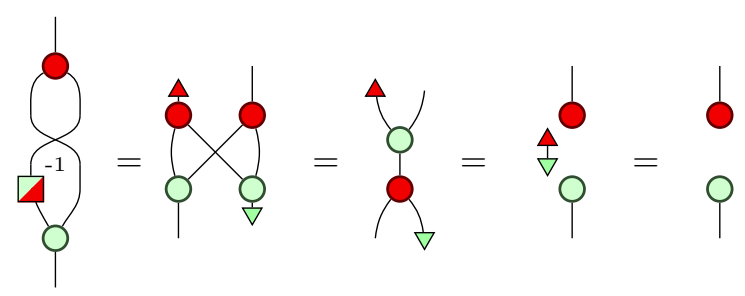

This implies that $H^{\sigma}$ is a Hopf algebra with $\nabla^{-1}$ as the antipode, and it follows from Proposition 2.11 that the antipode of $H^{\sigma}$ is the inverse of the antipode of $H$. However, for the sake of clarity we will replicate the proof. We show that $\boldsymbol{\nabla}^{-1} \circ \boldsymbol{\nabla}=1$ as follows

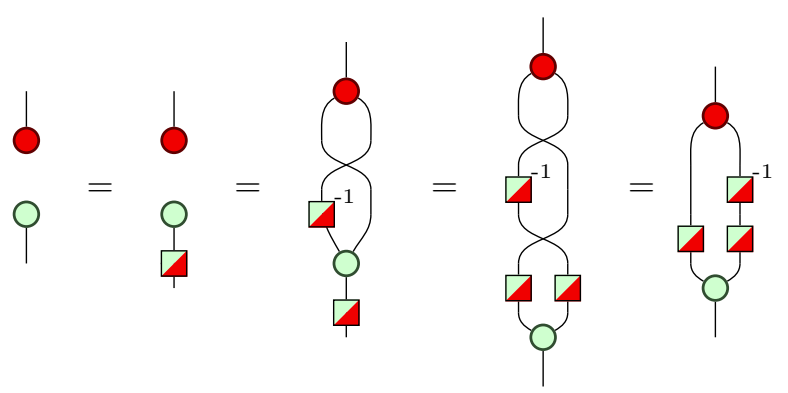

It follows from this that

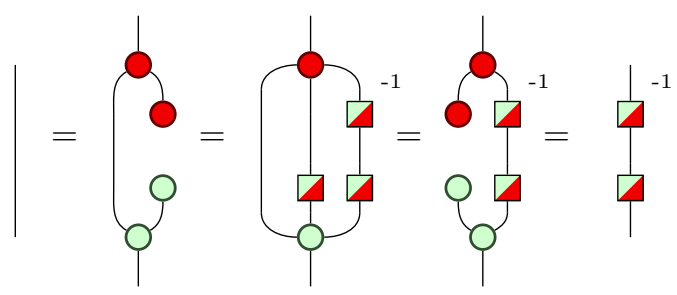

By a similar argument, $\boldsymbol{\nabla}^{-1} \circ \boldsymbol{\nabla}=1$. 


\section{Lemma A.1.}

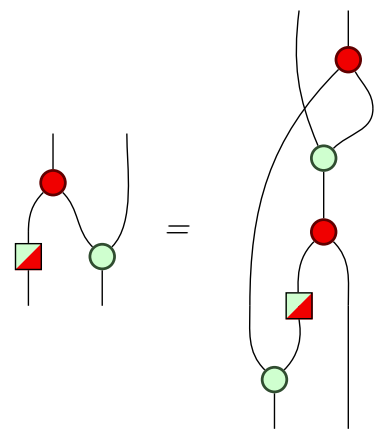

Proof. Observe that
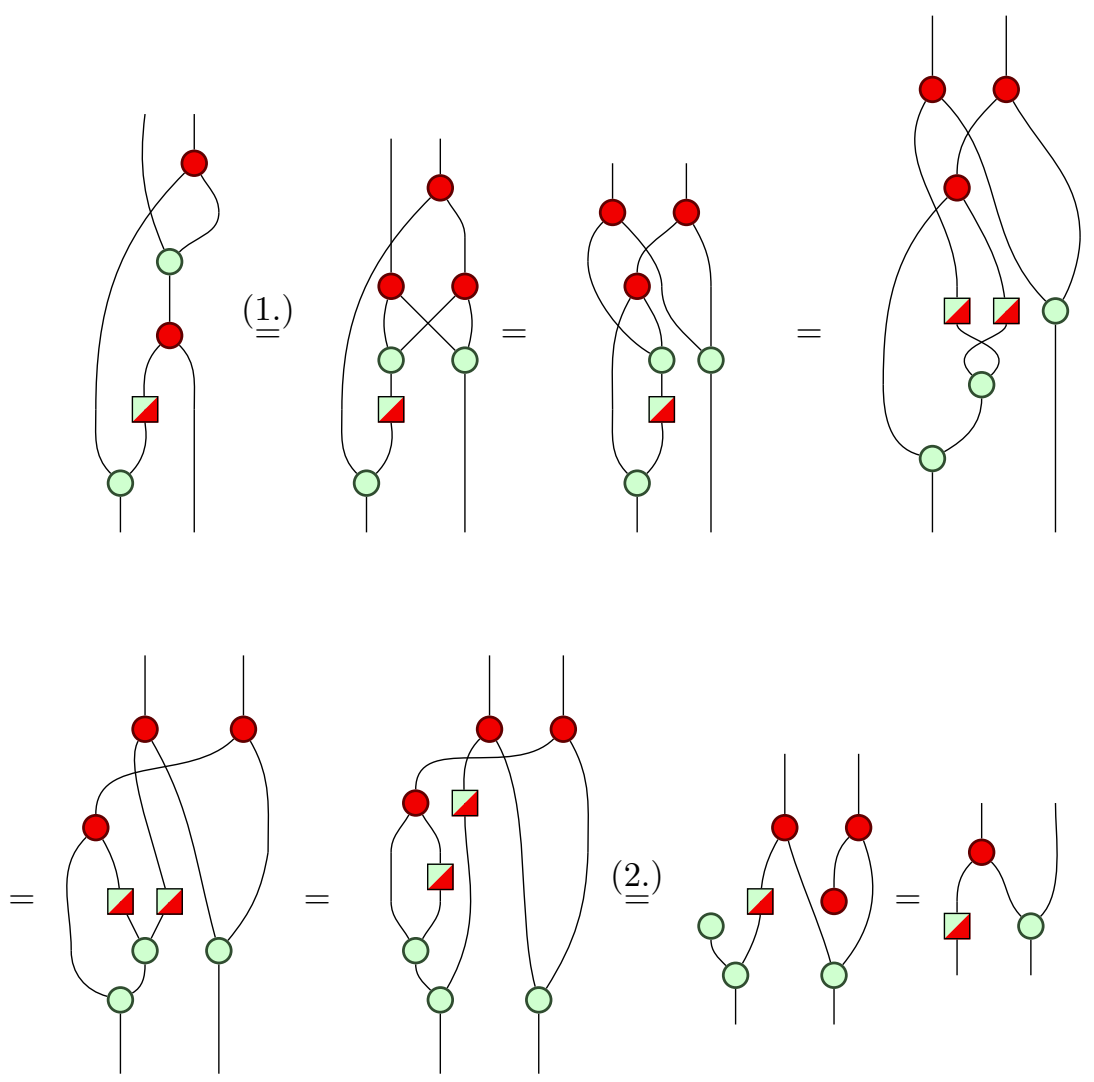

where (1.) comes from the bialgebra rule and (2.) comes from the Hopf law.

Lemma 3.8. Let $(H, \uparrow, \nabla)$ be an integral Hopf algebra. $\gamma$ is a right inverse for $\beta$ if and only if

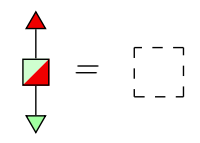


Proof. Suppose that $H$ is nondegenerate. Then

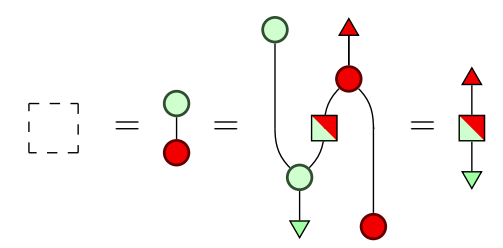

Consider the converse. Then we may characterise the unit as follows:

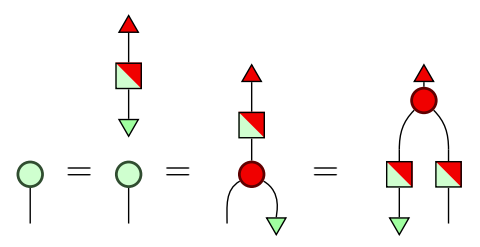

This implies that

$$
0=a_{\Delta^{-1}}=\sum_{\nabla}^{a}
$$

This then allows us to show the following, where (1.) comes from Lemma A.1, and (2.) is due to the definition of cointegrals.

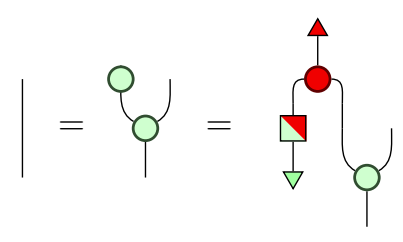

(1.)

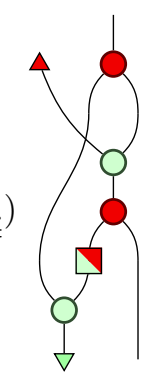

$(\underline{2 .})$

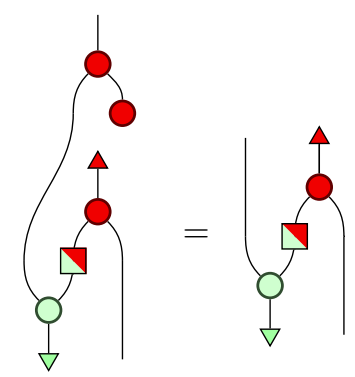

Hence, $H$ is nondegenerate, and we have our result.

Lemma 3.9. Let $(H, \uparrow, \downarrow)$ be an integral Hopf algebra. $H$ is nondegenerate if and only if $\beta$ is a Frobenius form for $(H, \mathcal{Y}, Q)$, or equivalently, if and only if $\gamma^{\prime}$ is a Frobenius form for $(H, \mathbf{Q}, \mathbf{O}) . H e n c e$, if $H$ is nondegenerate, then $H$ admits a pre-HF algebra structure.

Proof. If $H$ is nondegenerate, then the conditions of Definition 2.7 are satisfied.

Conversely, suppose that $\beta$ is a Frobenius form; then there exists some $\bar{\beta}$ such that

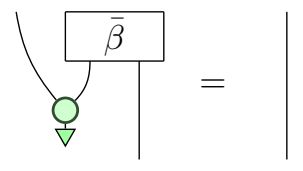


Appealing to Lemma 3.5 we have

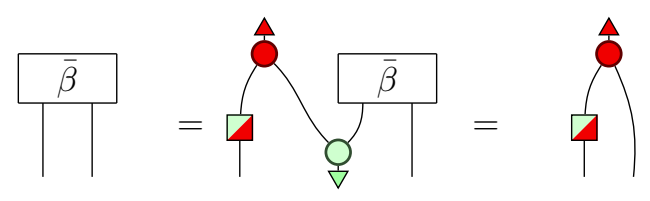

hence, $\gamma$ is the right inverse of $\beta$, and $H$ is nondegenerate. The proof for $\gamma^{\prime}$ is similar.

Lemma A.2. When $H$ has two half dual structures, $\cap, \forall$ and $\models, \forall$, then the integral morphisms coincide.

Proof.

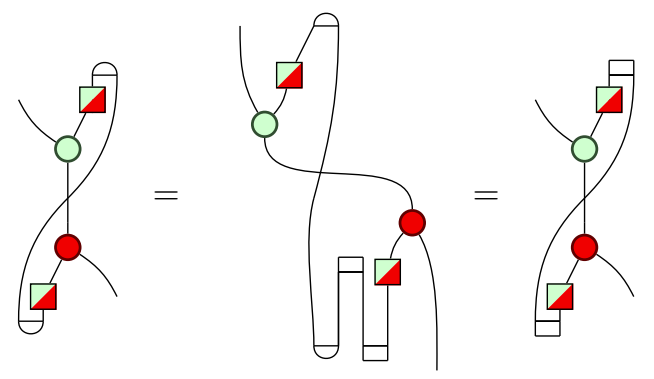

Lemma 3.11, Given a point $p: I \rightarrow H$, and copoint $q: H \rightarrow I$, the morphism $\mathcal{I} \circ p$ is a left cointegral, and $q \circ \mathcal{I}$ is a right integral. In addition, $p$ is a left cointegral if and only if $\mathcal{I} \circ p=p$, and $q$ is a right integral if and only if $q \circ \mathcal{I}=q$.

Proof. Out goal is to show that, for all points $p$,

$$
\underbrace{\frac{p}{\mathcal{I}}}_{\mathcal{I}}=\frac{p}{\mathcal{I}}
$$

If we are able to prove the following, then the result will follow.

$$
\left.\frac{1}{\mathcal{I}}\right)_{\mathcal{I}}=1
$$

As such, we may begin by composing $\mathcal{I}$ with ${ }^{\top}$ '. 


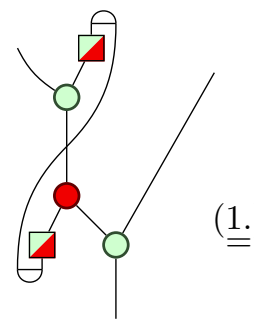

(1.)
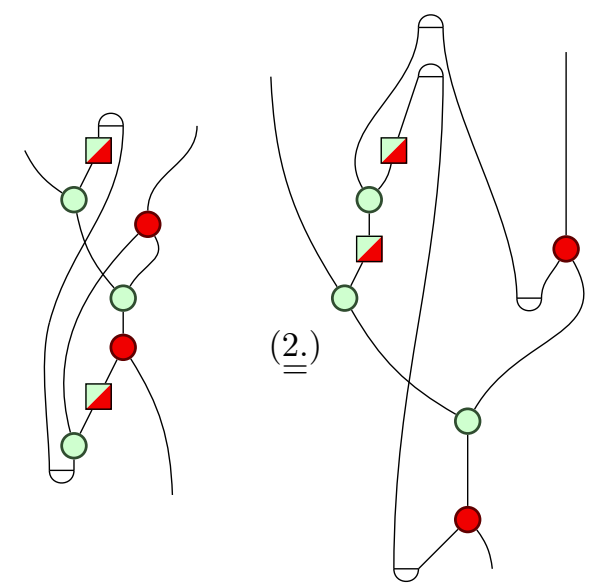

$(\underline{3 .})$

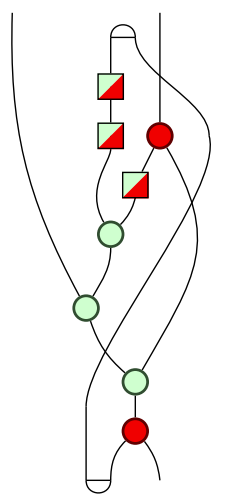

$(\underline{\underline{4} .})$

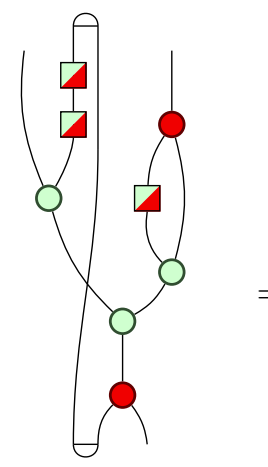

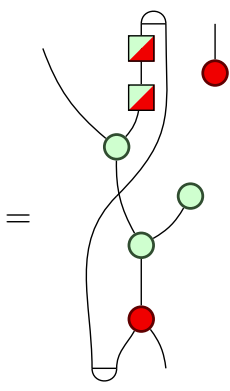

$(\underline{5 .})$

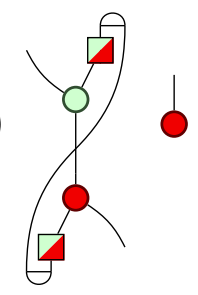

Where (1.) comes from Lemma A.1. (2.) comes from the presence of half duals. The antipode is a bialgebra homomorphism $H^{\mathrm{op}} \rightarrow H$, by Proposition 2.11, which gives us (3.). Associativity gives us (4.), and (5.) is due to the presence of half duals. Hence, we have proved our result.

Out result also tells us that if $\mathcal{I} \circ p=p$, then $p$ is a left cointegral. For the converse, let ${ }^{\boldsymbol{}}$ be a left cointegral. We then get

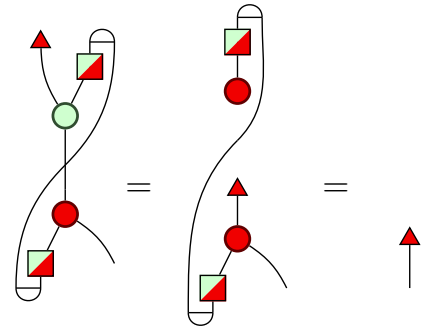

The proof for right integrals is similar.

Lemma 3.14. Let the object $H$ have a right half dual $H^{*}$, where $H$ is a Hopf algebra. $H$ fulfills the Frobenius condition if and only if there is an equaliser 4 of

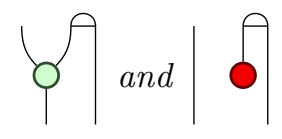


if and only if there is a coequaliser $\nabla$ of

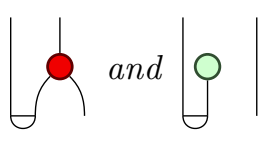

Proof. Suppose that $H$ fulfills the Frobenius condition. Then $(H, \uparrow, \downarrow)$ is an integral Hopf algebra by Theorem 3.13 , so

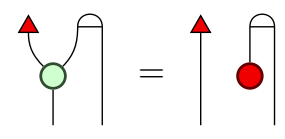

We shall actually prove that $\boldsymbol{\uparrow}$ is a split equaliser. To do so, we need to find a retract of $\boldsymbol{\Lambda}$ and . The Frobenius condition tells us that $\nabla$ is the retract of $\uparrow$, and we can easily calculate that the morphism is a retract of all we need to show now is

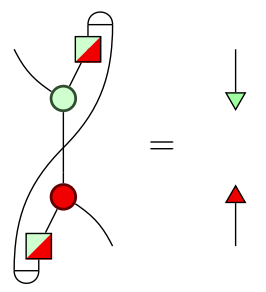

but this follows immediately from the assumption that the Frobenius condition is satisfied. Thus, we have one direction. Showing that $\dot{\nabla}$ is a split coequaliser follows dually.

For the other direction, note that by Lemma 3.11 we have

$$
\frac{1}{\mathcal{I}} \int_{0} \cap=\frac{1}{\mathcal{I}} \rho
$$

where $\mathcal{I}$ is the integral morphism. Thus, $\mathcal{I}$ is a cone of the appropriate diagram. We are assuming that $\boldsymbol{\Lambda}$ is an equaliser, so there is a unique morphism $\nabla: H \rightarrow I$ such that

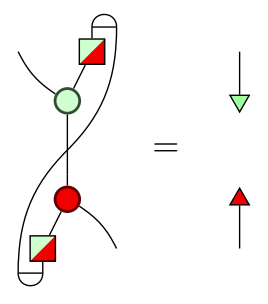

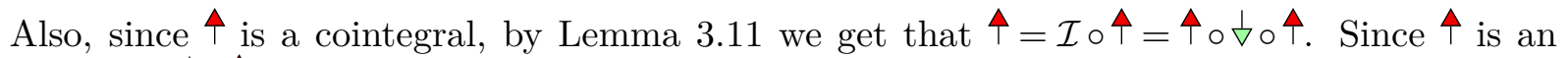
equaliser, $\nabla_{\circ} \hat{A}=1_{I}$. Hence, the Frobenius condition is satisfied. It is clear that if we assume that we have a coequaliser, $\nabla$, this will also imply the Frobenius condition by duality. 
Lemma 3.16. Let $(H, \uparrow, \nabla)$ be an integral Hopf algebra and suppose that $H$ has enough points. If every left cointegral (right integral) is a scalar multiple of $\mathbf{Q}$ (resp. $\mathrm{O}$ ) then $H$ fulfills the Frobenius condition

Proof. If we can show that

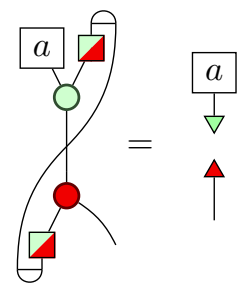

then, since $H$ has enough points, the result will follow. By Lemma 3.11, for all points $a, \mathcal{I} \circ a$ is a cointegral. Then, by hypothesis there exists a scalar $k: I \rightarrow I$ such that $\mathcal{I} \circ a=\boldsymbol{\Delta} \otimes k$

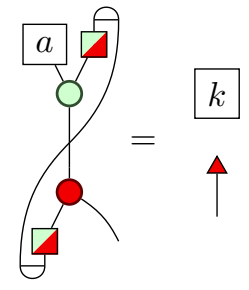

Hence, if we can show that $\nabla \circ a=k$, we will have our result. Observe that, since $\downarrow$ is an integral, $\nabla \circ \mathcal{I}=\downarrow$, so we get the following.

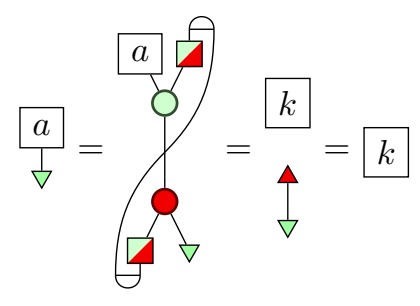

and the result follows.

Theorem 3.17. Let $H$ be a Hopf algebra such that the object $H$ has some weak right dual $H^{*}$. Then $H$ admits a Hopf-Frobenius algebra structure if and only if $H$ fulfills the Frobenius condition.

Proof. If $H$ is a Hopf-Frobenius algebra, then it admits a Frobenius algebra, and therefore, by Theorem 3.15, it fulfills the Frobenius condition.

Consider the converse. In what follows, we will prove the Theorem by first proving some intermediary lemmas. If $H$ fulfills the Frobenius condition, then this is equivalent, by Theorem 3.13 to $H$ admitting a pre-HF structure such that $(H, ९$, ○) is an integral Hopf algebra. We begin by proving that $\nabla=\Omega$.

Lemma A.3. Let $H$ admit a pre-HF algebra structure; $(H, \emptyset, \bigcirc)$ is an integral Hopf algebra if and only if $\boldsymbol{\nabla}=$ R. 
Proof. The implication in one direction follows from Lemma 3.5. Suppose the converse. Note that $\nabla=\Omega$ is equivalent to $\nabla^{-1}=\Omega$. We use the fact that the antipode is a bialgebra homomorphism to get the following.

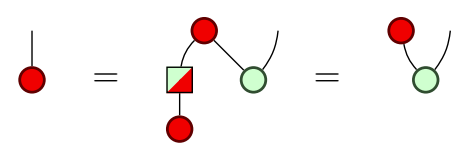

It follows from this that that we may express 9 similarly.

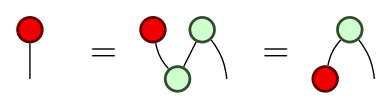

This allows us to show that $\varphi$ is a left cointegral.

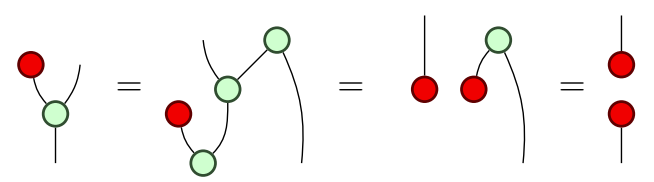

The proof that $\circlearrowleft$ is a right integral is similar. We only need to show that $\circlearrowleft \circ \varphi_{\mathrm{P}}=1_{I}$, but this follows from above.

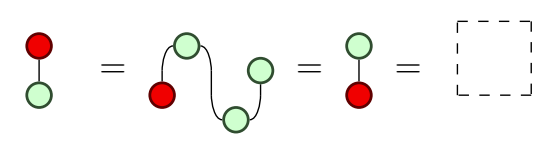

It follows immediately from this Lemma that when $H$ fulfills the Frobenius condition, the antipode is the canonical isomorphism that maps from one dual structure to the other, in the sense of Proposition C.1. We record this fact as a Corollary.

Corollary A.4. Let $H$ admit a pre-HF algebra structure, such that $(H, \bigcirc, \bigcirc)$ is an integral Hopf algebra. Then

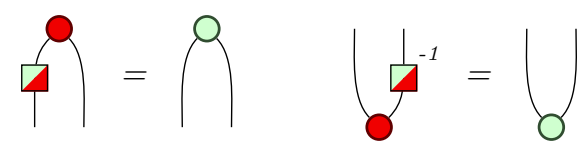

To prove the Theorem, we must show that $(H, \boldsymbol{\gamma}, \boldsymbol{\varphi}, \boldsymbol{\alpha}, \boldsymbol{\phi}, \nabla)$ forms a Hopf algebra, where $\boldsymbol{\nabla}=\boldsymbol{\gamma}$. We will accomplish this by showing first that $(H, \boldsymbol{\gamma}, \boldsymbol{\phi}, \boldsymbol{\alpha}, \boldsymbol{\phi})$ forms a bialgebra, and then that $\boldsymbol{\nabla}$ is the appropriate antipode. Recall that the dual of a Hopf algebra is a Hopf algebra, in the sense of Definition 2.12. By using the dual structure of the green Frobenius algebra, we get the following:

Lemma A.5. Let $H$ admit a pre-HF algebra structure such that $(H, \emptyset, \circlearrowleft)$ is an integral Hopf algebra, and let $(\cdot)^{\circ}$ be the duality defined by the green Frobenius algebra (cf. Lemma 2.6). Then:

$$
(0)^{\circ}=h \quad(9)^{\circ}=b \quad(q)^{\circ}=\varnothing \quad(b)^{\circ}=9
$$


Proof. The first two statements are clear from the definition of the green dual. For the third statement, we see that

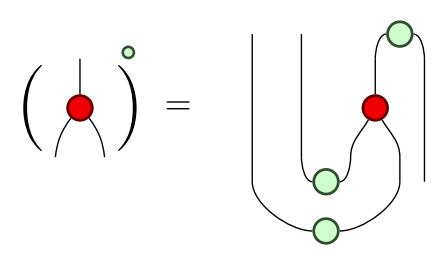

$(\underline{\underline{1}})$

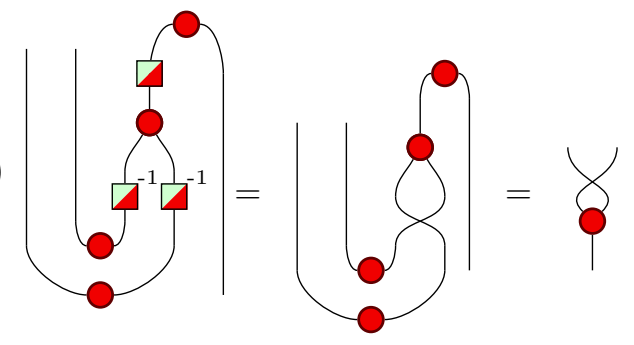

where (1.) comes from Corollary A.4. The final statement follows from above, as $(\boldsymbol{\phi})^{\circ}$ will be the unit of $(\boldsymbol{\alpha})^{\circ}$. Units of monoids are unique, so $(\boldsymbol{\phi})^{\circ}=\boldsymbol{\varphi}$.

We now have that $H^{\circ}:=\left(H, \mathbf{Q}^{\circ}, \mathbf{0}^{\circ}, \mathbf{\gamma}^{\circ}, \mathrm{Q}^{\circ}, \mathbf{\square}^{\circ}\right)$ is a Hopf algebra. By the above Lemma,

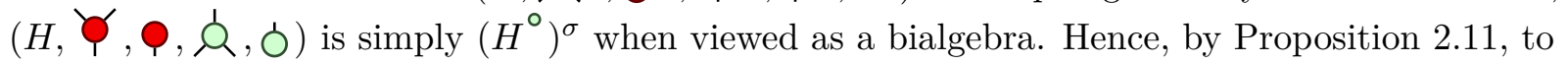
show that this is a Hopf algebra, we only need to show that $\square^{\circ}$ is invertible, and that it is equal to ${ }^{\circ}$. But $(\cdot)^{\circ}$ preserves inverses, so we know that $\boldsymbol{\nabla}=\left(\nabla^{\circ}\right)^{-1}$. All that remains is showing that $\boldsymbol{\nabla}$ has the appropriate form, and this is done by straightforward calculation.

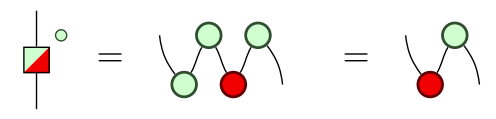

Hence, $\boldsymbol{\nabla}=\left(\boldsymbol{\nabla}^{\circ}\right)^{-1}={ }^{\circ}$. Therefore, if $H$ fulfills the Frobenius condition, the $H$ admits a Hopf-Frobenius algebra structure.

Corollary A.6. Let $H$ admit a pre-HF algebra structure, such that $(H, \bigcirc, \bigcirc)$ is an integral Hopf algebra. Then

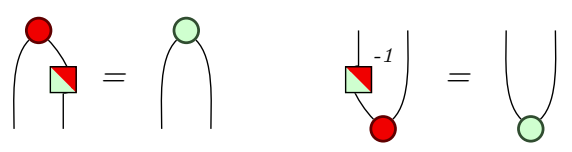

Lemma 3.19, Let $H$ admit a Hopf-Frobenius algebra structure. Then this structure is unique up to invertible scalar.

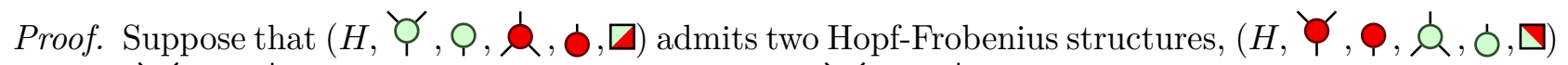

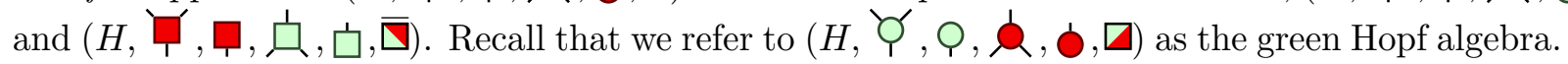
The Hopf-Frobenius structures share a green Hopf algebra, so the respective units and counits of these Hopf algebras must be left cointegrals and right integrals of the green Hopf algebra. It follows from Lemma 3.14 that there exists unique scalars, $k, l: I \rightarrow I$, such that $\varphi=\square \otimes k$ and

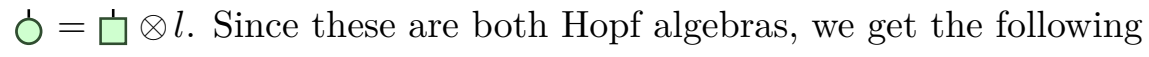

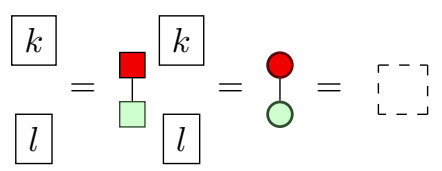


Thus, $k$ and $l$ are mutually inverse. We now only need to show that the other structure maps are scalar multiples of each other. We see that $\bar{\nabla}=\boldsymbol{\nabla}$ follows from the above proof, as

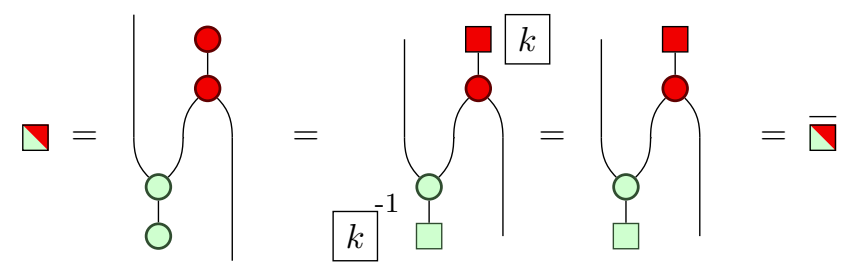

Finally, this will imply that the multiplication and comultiplication maps only differ by an invertible scalar. Note that as $\bar{\nabla}=\boldsymbol{\nabla}$, their inverses will also coincide. Recall how constructed, and observe that

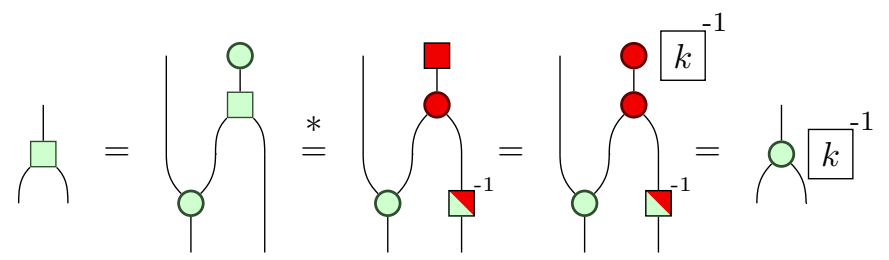

where $\left(^{*}\right)$ comes from Corollary A.6. The same is true for $\Psi^{\top}$ and ${ }^{\prime}{ }^{\prime}$. Hence, if $H$ admits two Hopf-Frobenius algebra structures, then they will only differ by an invertible scalar factor.

Lemma 5.2. The morphism $\square$ is a Hopf algebra homomorphism between $H_{\bullet}^{\sigma}$ and $H_{\circ}^{*}$.

Proof. We will only show that $\square$ is a homomorphism for $\dot{\alpha}^{*}$, the rest of the structure maps will have similar proofs. We first note that, by Corollary A.4

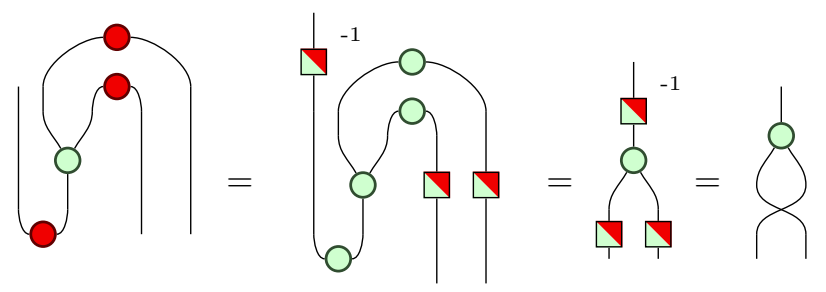

Hence, we see that

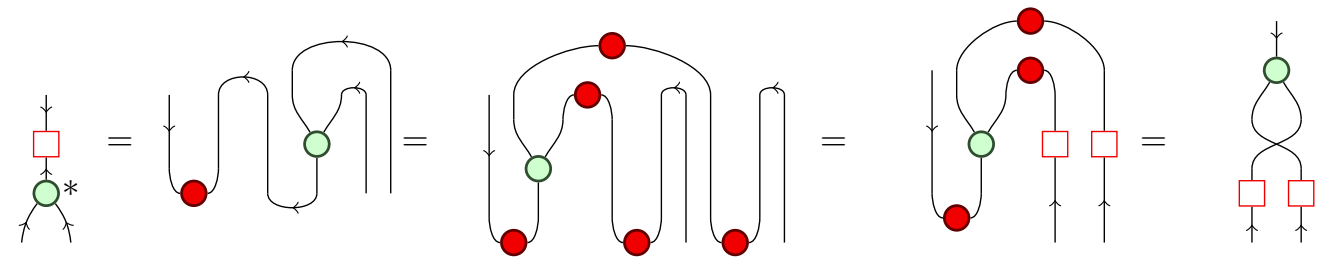




\section{Lemma 5.8.}

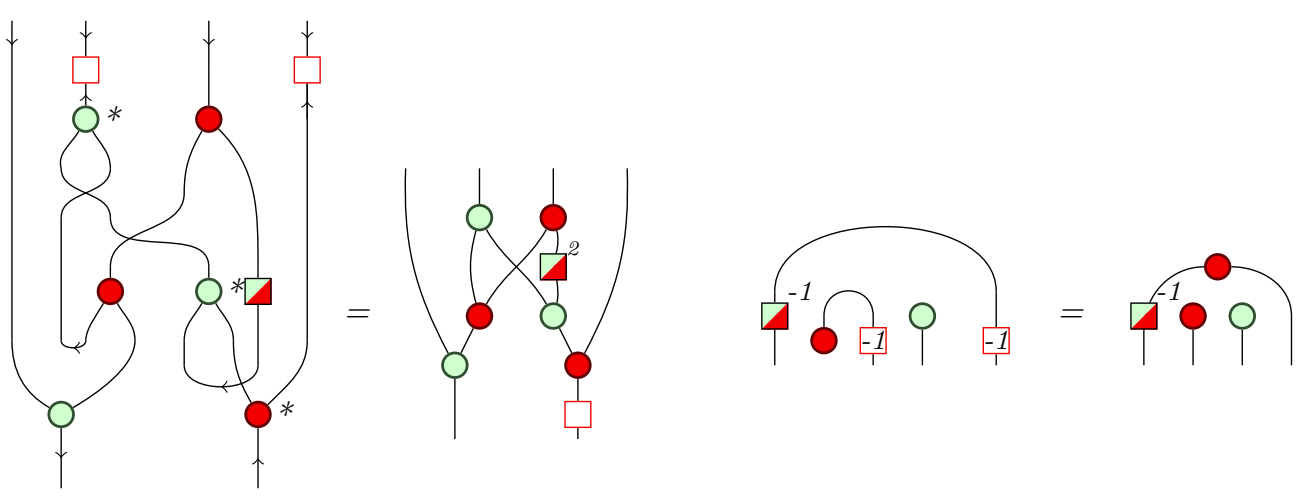

Proof. This is clear from the definition of $\square$, Lemma 5.2 and Corollary A.4. We explicitly spell out the first statement here.

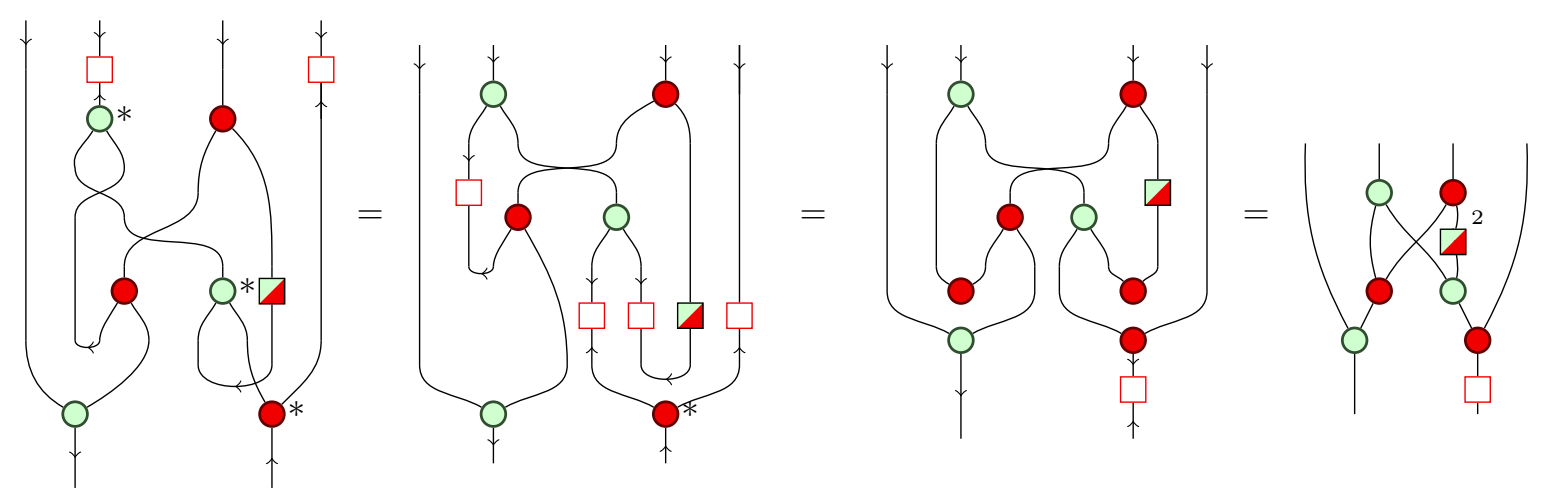

The proof of the second statement follows immediately from the definition of 11 .

\section{B Taft Hopf algebra for $n=2$}

Here we shall state the Hopf-Frobenius algebra for the 4 dimensional Taft Hopf algebra explicitly. It is generated by $g$ and $x$, and has the structure

\begin{tabular}{|c|c|c|c|c|c|c|c|c|c|c|}
\hline Y & 1 & $x$ & $g$ & $g x$ & a & & 0 & & $\nabla$ & \\
\hline 1 & 1 & $x$ & $g$ & $g x$ & 1 & $1 \otimes 1$ & 1 & 1 & 1 & 1 \\
\hline$x$ & $x$ & 0 & $-g x$ & 0 & $x$ & $1 \otimes x+x \otimes g$ & $x$ & 0 & $x$ & $g x$ \\
\hline$g$ & $g$ & $g x$ & 1 & $x$ & $g$ & $g \otimes g$ & $g$ & 1 & $g$ & $g$ \\
\hline$g x$ & $g x$ & 0 & $-x$ & 0 & $g x$ & $g \otimes g x+g x \otimes 1$ & $g x$ & 0 & $g x$ & $-x$ \\
\hline
\end{tabular}

FVect $_{\mathbf{k}}$ is a compact closed category, so the integral projection is the map

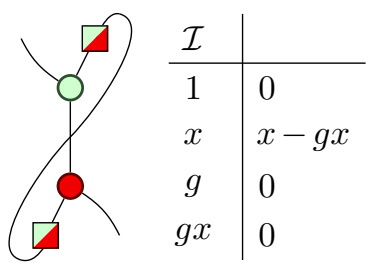


Hence, the element $x-g x$ is a left cointegral, and the right integral is the delta function for $x$, $\delta_{x}$. Hence, by Theorem 3.13 , these shall be our unit and counit respectively. It is now possible to construct the resulting Hopf-Frobenius algebra, but we shall explicitly state the structure maps. The green Frobenius algebra is

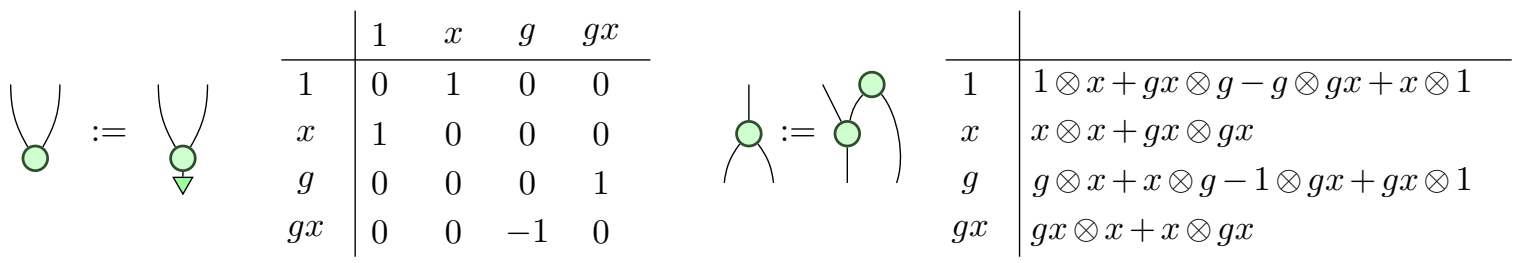

$$
\begin{aligned}
& \bigcap:=\{=1 \otimes x+g x \otimes g-g \otimes g x+x \otimes 1
\end{aligned}
$$

and the red Frobenius algebra

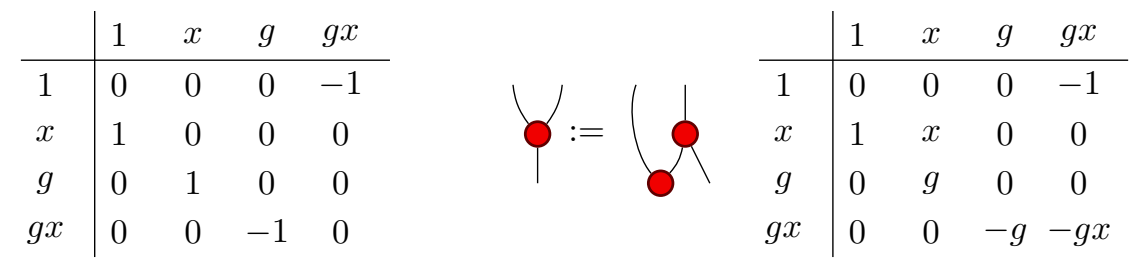

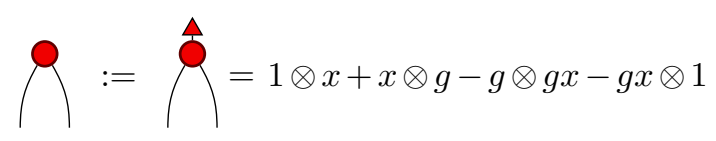

\section{Additional Background Material}

In this section we provide additional definitions and basic properties to flesh out the background material of Section 2 .

\section{C.1 Categories with duals}

Proposition C.1. In a monoidal category $\mathcal{C}$ suppose that $A$ has two right duals $\left(B_{1}, d_{1}, e_{1}\right)$ and $\left(B_{2}, d_{2}, e_{2}\right)$; then there exists an isomorphism $f: B_{1} \cong B_{2}$, satisfying the equations shown below.

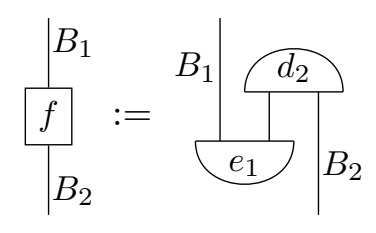
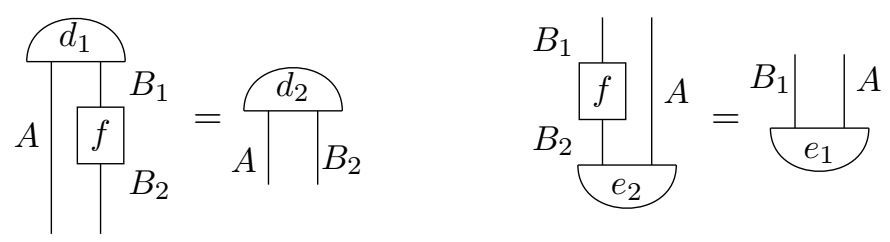

Proof. Define $f$ as shown above; the required equations follow immediately.

\section{C.2 Monoids and Comonoids}

Definition C.2. A monoid in a monoidal category $\mathcal{C}$ consists of an object $M$, a binary multiplication $\mu: M \otimes M \rightarrow M$ and a unit morphism $\eta: I \rightarrow M$ obeying the familiar associativity and 
unit laws, shown in diagram form below.
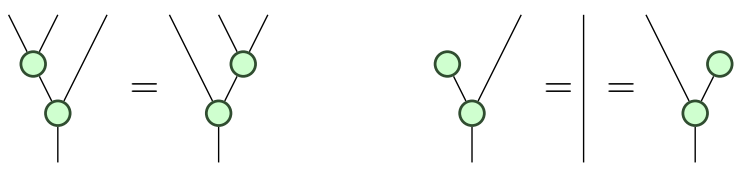

A comonoid in $\mathcal{C}$ is a monoid in $\mathcal{C}^{\text {op }}$, concretely depicted below.
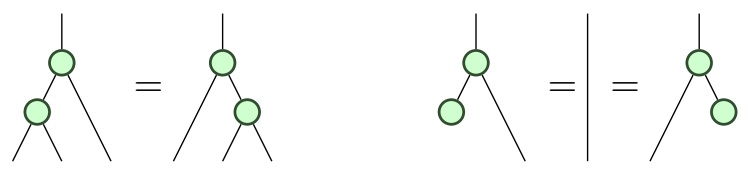

A (co)monoid is called (co)commutative if its (co)multiplication is invariant under the exchange map, as depicted below.

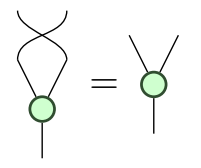

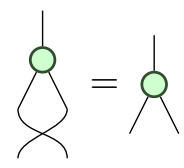

In this paper we will not assume commutativity or cocommutativity.

Definition C.3. Given a monoid ( $\left.M,{ }^{\prime}, \mathrm{Y}, \mathrm{Q}\right)$, a point $a: I \rightarrow M$ is left invertible if there exists a point $l: I \rightarrow M$ satisfying the left equation below; it is right invertible if there exists $r: I \rightarrow M$ satisfying the right equation; it is invertible if it is both left and right invertible, in which case the two inverses coincide.

$$
\frac{\widehat{A} \hat{a}}{\hat{i}}=9=\frac{\widehat{a} \hat{r}}{0}
$$

Co-invertibility of co-points $\alpha: M \rightarrow I$ with respect to a comonoid is defined dually.

\section{C.3 Frobenius algebras}

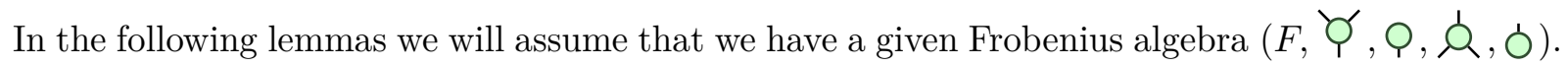

Definition C.4. A Frobenius algebra is called symmetric if its cap (or equivalently its cup) is invariant under the symmetry.
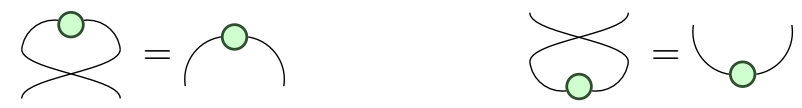

Proof. See Kock [23].

Lemma C.5. There is a bijective correspondence between invertible points for the monoid and coinvertible copoints for the comonoid.

Proof. Let $(\cdot)^{\circ}$ be the duality induced by the cup and cap; then $u: I \rightarrow F$ is invertible iff and only if $u^{\circ}: F \rightarrow I$ is coinvertible. 
Lemma C.6. Let $u$ be a coinvertible element of the comonoid. Define<smiles></smiles>

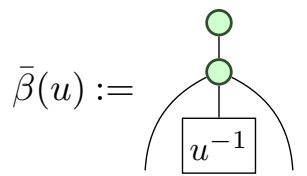

Then $\beta(u)$ is a Frobenius form for the monoid $\left(F, \mathcal{Y}^{\prime}, \mathrm{Q},\right)$.

Proof. We must show that the equations of 2.7 hold. The first follows from associativity of the monoid. For the second we have:

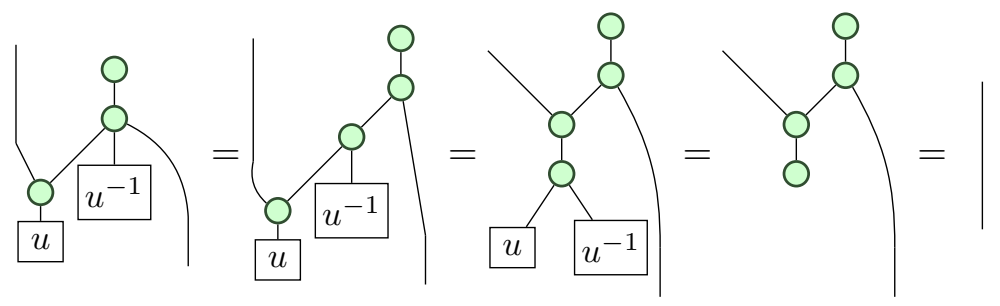

and similarly for the other side. Note that $\beta(u)=\beta(v)$ implies $u=v$ by the uniqueness of inverses.

Lemma C.7. Suppose that $\beta$ is a Frobenius form on 'Y'; then we obtain a coinvertible element $u: F \rightarrow I$ as follows:

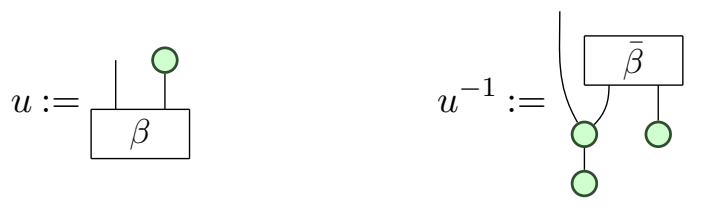

Proof. We need only to show that $u^{-1}$ is the coinverse of $u$.

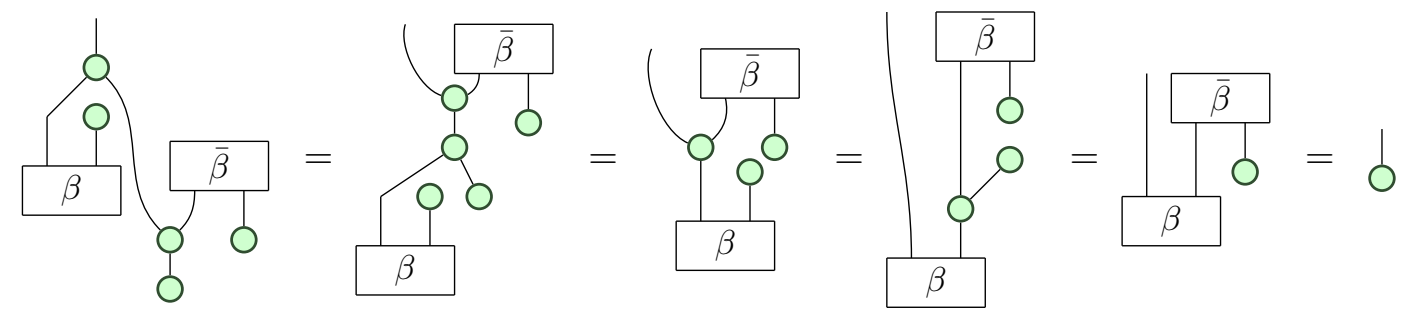

Combining the three preceding lemmas we obtain:

Proposition C.8. There is a bijective correspondence between the invertible elements of a monoid and the Frobenius forms definable on it. 